\title{
Development of Bisindole-Substituted Aminopyrazoles as Novel GSK-3 $\beta$ Inhibitors with Suppressive Effects against Microglial Inflammation and Oxidative Neurotoxicity
}

\author{
Jian-Guo Liu, ${ }^{a, c, \#}$ Danfeng Zhao, ${ }^{b, \#}$ Qi Gong, ${ }^{\mathrm{b}}$ Fengxia Bao, ${ }^{b}$ Wen-Wen Chen,${ }^{c, *}$ Haiyan Zhang, ${ }^{b, *}$ \\ and Ming-Hua Xu ${ }^{a, c, *}$ \\ ${ }^{a}$ Shenzhen Key Laboratory of Small Molecule Drug Discovery and Synthesis, Department of \\ Chemistry, Southern University of Science and Technology, 1088 Xueyuan Boulevard, Shenzhen \\ 518055, China \\ ${ }^{b}$ CAS Key Laboratory of Receptor Research, Shanghai Institute of Materia Medica, Chinese \\ Academy of Sciences, 555 Zuchongzhi Road, Shanghai 201203, China \\ ${ }^{c}$ State Key Laboratory of Drug Research, Shanghai Institute of Materia Medica, Chinese \\ Academy of Sciences, 555 Zuchongzhi Road, Shanghai 201203, China \\ E-mail: wenwen@shnu.edu.cn, hzhang@simm.ac.cn, xumh@sustech.edu.cn
}

\section{Table of contents}

1. Characterization data of synthetic intermediates $11,14,18$ and 25

2. Copies of NMR spectra of aminopyrazoles 4a-d, 5a-d, 6a-m and 7a-c

3. Cytotoxic effects of compounds $\mathbf{6 c}, \mathbf{6 e}, \mathbf{6 h}, 7 \mathbf{a}$ and 7c on BV-2 and HT-22 cells 


\section{Characterization data of synthetic intermediates $11,14,18$ and 25}

2-(1-methyl-1H-indol-3-yl)-3-oxo-3-phenylpropanenitrile (11a).

${ }_{3 \mathrm{H}) .}^{1} \mathrm{H} \mathrm{NMR}(300 \mathrm{MHz}$, Chloroform- $d) \delta 7.98(\mathrm{~d}, J=7.2,2 \mathrm{H}), 7.71(\mathrm{~d}$,
$J=7.8,1 \mathrm{H}), 7.61-7.17(\mathrm{~m}, 7 \mathrm{H}), 7.13(\mathrm{~s}, 1 \mathrm{H}), 5.87(\mathrm{~s}, 1 \mathrm{H}), 3.74(\mathrm{~s}$,

2-(1-methyl-1H-indol-3-yl)-3-oxo-3-(p-tolyl)propanenitrile (11b)

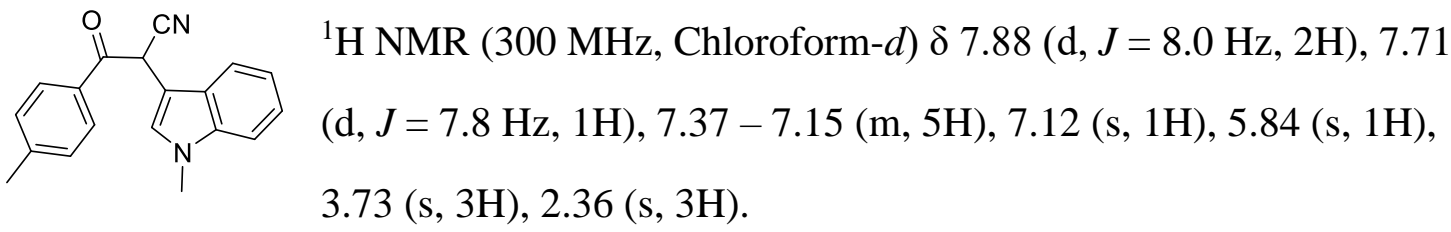

2-(1-methyl-1H-indol-3-yl)-3-oxo-3-(m-tolyl)propanenitrile (11c)

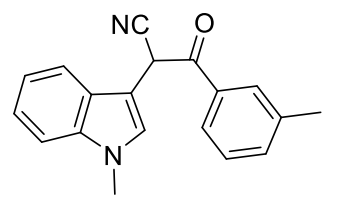

${ }^{1} \mathrm{H}$ NMR (300 MHz, Chloroform- $d$ ) $\delta 7.88-7.64$ (m, 3H), $7.44-$

$7.16(\mathrm{~m}, 5 \mathrm{H}), 7.13(\mathrm{~s}, 1 \mathrm{H}), 5.87(\mathrm{~s}, 1 \mathrm{H}), 3.74(\mathrm{~s}, 3 \mathrm{H}), 2.35$ (s, $3 \mathrm{H})$.

3-(3-bromophenyl)-2-(1-methyl-1H-indol-3-yl)-3-oxopropanenitrile (11d)

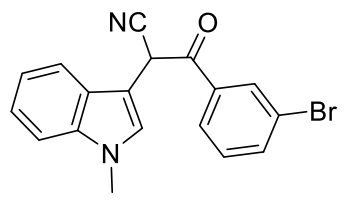

${ }^{1} \mathrm{H}$ NMR (300 MHz, Chloroform- $\left.d\right) \delta 8.12(\mathrm{~d}, J=1.7 \mathrm{~Hz}, 1 \mathrm{H})$, $7.91-7.81(\mathrm{~m}, 1 \mathrm{H}), 7.72-7.59(\mathrm{~m}, 3 \mathrm{H}), 7.44-7.17(\mathrm{~m}, 6 \mathrm{H})$, $7.13(\mathrm{~s}, 1 \mathrm{H}), 5.80(\mathrm{~s}, 1 \mathrm{H}), 3.76(\mathrm{~s}, 3 \mathrm{H})$.

3-(1-methyl-1H-indol-3-yl)-3-oxo-2-phenylpropanenitrile (14a)

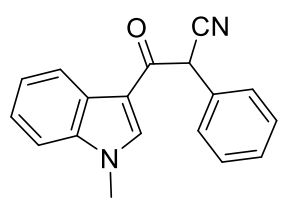

${ }^{1} \mathrm{H}$ NMR (300 MHz, Chloroform- $d$ ) $\delta 8.40-8.30(\mathrm{~m}, 1 \mathrm{H}), 7.80(\mathrm{~s}$, 1H), $7.56-7.45(\mathrm{~m}, 2 \mathrm{H}), 7.42-7.28(\mathrm{~m}, 6 \mathrm{H}), 5.27(\mathrm{~s}, 1 \mathrm{H}), 3.82(\mathrm{~s}$, $3 \mathrm{H})$. 
${ }^{1} \mathrm{H}$ NMR (300 MHz, Chloroform- $d$ ) $\delta 7.85$ (d, $\left.J=2.5 \mathrm{~Hz}, 1 \mathrm{H}\right), 7.75$ (s, 1H), 7.50 (dd, (n) $J=7.9,1.7 \mathrm{~Hz}, 2 \mathrm{H}), 7.42-7.31(\mathrm{~m}, 3 \mathrm{H}), 7.20(\mathrm{~d}, J=8.9 \mathrm{~Hz}$, 1H), $6.95(\mathrm{dd}, J=8.9,2.5 \mathrm{~Hz}, 1 \mathrm{H}), 5.26(\mathrm{~s}, 1 \mathrm{H}), 3.86(\mathrm{~d}, J=1.0$ $\mathrm{Hz}, 3 \mathrm{H}), 3.79$ (d, $J=1.0 \mathrm{~Hz}, 3 \mathrm{H})$.

3-(5-bromo-1-methyl-1H-indol-3-yl)-3-oxo-2-phenylpropanenitrile (14c)

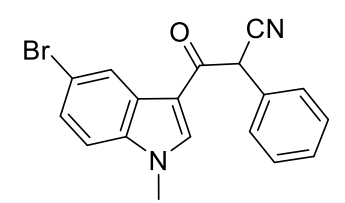

${ }^{1} \mathrm{H}$ NMR (300 MHz, Chloroform- $d$ ) $\delta 8.51(\mathrm{~d}, J=1.9 \mathrm{~Hz}, 1 \mathrm{H})$, $7.79(\mathrm{~s}, 1 \mathrm{H}), 7.59-7.29(\mathrm{~m}, 6 \mathrm{H}), 7.18(\mathrm{~d}, J=8.7 \mathrm{~Hz}, 1 \mathrm{H}), 5.22$ $(\mathrm{s}, 1 \mathrm{H}), 3.82(\mathrm{~s}, 3 \mathrm{H})$.

3-(1-methyl-1H-indol-3-yl)-3-oxo-2-(o-tolyl)propanenitrile (14d)

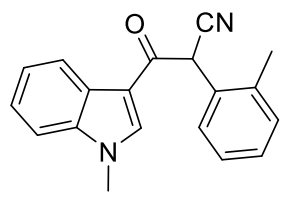

${ }^{1} \mathrm{H}$ NMR (300 MHz, Chloroform- $d$ ) $\delta 8.40-8.30$ (m, 1H), 7.68 (s, 1H), $7.53(\mathrm{dd}, J=6.4,2.5 \mathrm{~Hz}, 1 \mathrm{H}), 7.39-7.15(\mathrm{~m}, 6 \mathrm{H}), 5.41(\mathrm{~s}$, $1 \mathrm{H}), 3.80(\mathrm{~s}, 3 \mathrm{H}), 2.38(\mathrm{~s}, 3 \mathrm{H})$.

2,3-bis(1-methyl-1H-indol-3-yl)-3-oxopropanenitrile (18a)

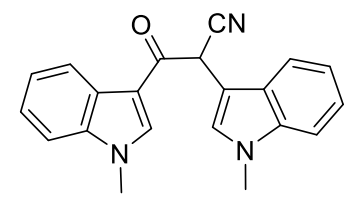

1H NMR (300 MHz, Chloroform-d) $\delta 8.44-8.32(\mathrm{~m}, 1 \mathrm{H})$, $7.82(\mathrm{~s}, 1 \mathrm{H}), 7.72(\mathrm{~d}, J=7.9 \mathrm{~Hz}, 1 \mathrm{H}), 7.37-7.12(\mathrm{~m}, 7 \mathrm{H})$, $5.53(\mathrm{~s}, 1 \mathrm{H}), 3.76(\mathrm{~s}, 3 \mathrm{H}), 3.73(\mathrm{~s}, 3 \mathrm{H})$.

3-(4-fluoro-1-methyl-1H-indol-3-yl)-2-(1-methyl-1H-indol-3-yl)-3-oxopropanenitrile $(18 b)$

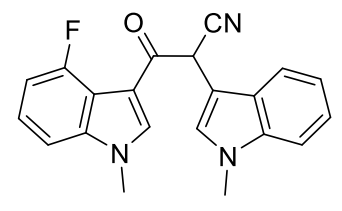

${ }^{1} \mathrm{H}$ NMR (300 MHz, DMSO-d6) $\delta 8.57$ (s, 1H), 8.07 (dd, $J=$ $8.5,0.5 \mathrm{~Hz}, 1 \mathrm{H}), 7.84(\mathrm{dd}, J=1.8,0.5 \mathrm{~Hz}, 1 \mathrm{H}), 7.76(\mathrm{ddd}, J=$ 7.8, 1.3, 0.8 Hz, 1H), 7.58 (s, 1H), $7.47-7.33$ (m, 2H), 7.18 (ddd, $J=8.2,7.0,1.3 \mathrm{~Hz}, 1 \mathrm{H}), 7.10$ (ddd, $J=8.1,7.0,1.1 \mathrm{~Hz}, 1 \mathrm{H}), 6.39$ (s, 1H), 3.84 (s, 3H), $3.76(\mathrm{~s}, 3 \mathrm{H})$. 
3-(5-fluoro-1-methyl-1H-indol-3-yl)-2-(1-methyl-1H-indol-3-yl)-3-oxopropanenitrile (18c)

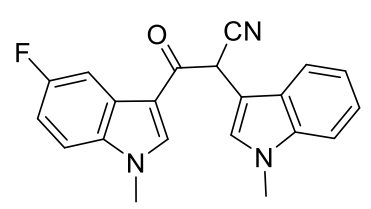

${ }^{1} \mathrm{H}$ NMR (300 MHz, Chloroform- $\left.d\right) \delta 8.06(\mathrm{~d}, J=2.6 \mathrm{~Hz}, 1 \mathrm{H})$, $7.83(\mathrm{~s}, 1 \mathrm{H}), 7.71(\mathrm{dt}, J=7.8,1.0 \mathrm{~Hz}, 1 \mathrm{H}), 7.32-7.24(\mathrm{~m}, 2 \mathrm{H})$, $7.22-7.15(\mathrm{~m}, 3 \mathrm{H}), 7.03(\mathrm{td}, J=8.9,2.6 \mathrm{~Hz}, 1 \mathrm{H}), 5.48(\mathrm{~s}, 1 \mathrm{H})$, $3.74(\mathrm{~s}, 3 \mathrm{H}), 3.74(\mathrm{~s}, 3 \mathrm{H})$.

3-(6-fluoro-1-methyl-1H-indol-3-yl)-2-(1-methyl-1H-indol-3-yl)-3-oxopropanenitrile (18d)

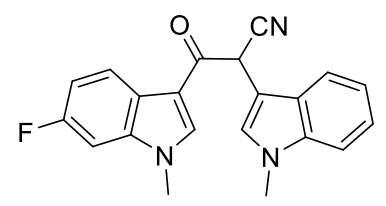

${ }^{1} \mathrm{H}$ NMR (300 MHz, Chloroform- $d$ ) $\delta 8.32(\mathrm{~d}, J=8.8 \mathrm{~Hz}$, 1H), $7.83(\mathrm{~s}, 1 \mathrm{H}), 7.70(\mathrm{~d}, J=7.9 \mathrm{~Hz}, 1 \mathrm{H}), 7.38-7.14(\mathrm{~m}$, 4H), $7.11-7.01(\mathrm{~m}, 1 \mathrm{H}), 6.97(\mathrm{dd}, J=9.2,2.3 \mathrm{~Hz}, 1 \mathrm{H}), 5.52$ (s, 1H), $3.76(\mathrm{~s}, 3 \mathrm{H}), 3.73$ (s, 3H).

3-(5-bromo-1-methyl-1H-indol-3-yl)-2-(1-methyl-1H-indol-3-yl)-3-oxopropanenitrile $(18 \mathbf{e})$

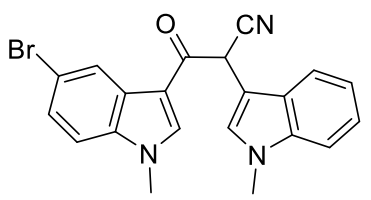

${ }^{1} \mathrm{H}$ NMR (300 MHz, Chloroform- $d$ ) $\delta 8.48(\mathrm{~d}, J=2.0 \mathrm{~Hz}$, $1 \mathrm{H}), 7.73(\mathrm{~s}, 1 \mathrm{H}), 7.33-7.21(\mathrm{~m}, 4 \mathrm{H}), 7.20-7.13(\mathrm{~m}, 2 \mathrm{H})$, $7.05(\mathrm{~d}, J=8.7 \mathrm{~Hz}, 1 \mathrm{H}), 5.45(\mathrm{~s}, 1 \mathrm{H}), 3.71(\mathrm{~s}, 3 \mathrm{H}), 3.69(\mathrm{~s}$, $3 \mathrm{H})$.

3-(6-bromo-1-methyl-1H-indol-3-yl)-2-(1-methyl-1H-indol-3-yl)-3-oxopropanenitrile (18f)

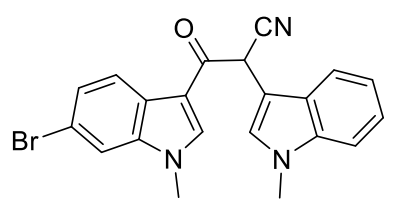

${ }^{1} \mathrm{H}$ NMR (300 MHz, DMSO-d6) $\delta 8.57(\mathrm{~s}, 1 \mathrm{H}), 8.06(\mathrm{~d}, J=$ $8.5 \mathrm{~Hz}, 1 \mathrm{H}), 7.84(\mathrm{~d}, J=1.7 \mathrm{~Hz}, 1 \mathrm{H}), 7.76(\mathrm{dt}, J=7.8,1.1 \mathrm{~Hz}$, 1H), $7.58(\mathrm{~s}, 1 \mathrm{H}), 7.46-7.35$ (m, 2H), 7.14 (dddd, $J=24.6$, 8.1, 7.0, 1.2 Hz, 2H), 6.39 (s, 1H), 3.85 (s, 3H), 3.76 (s, 3H). 
3-(7-bromo-1-methyl-1H-indol-3-yl)-2-(1-methyl-1H-indol-3-yl)-3-oxopropanenitrile $(18 g)$

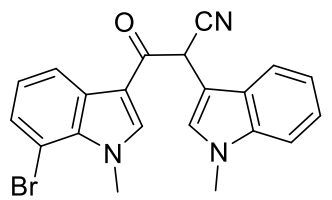

${ }^{1} \mathrm{H}$ NMR (300 MHz, Chloroform- $d$ ) $\delta 8.38(\mathrm{dd}, J=8.0,1.1 \mathrm{~Hz}$, 1H), $7.77(\mathrm{~s}, 1 \mathrm{H}), 7.73-7.68(\mathrm{~m}, 1 \mathrm{H}), 7.42(\mathrm{dd}, J=7.7,1.0 \mathrm{~Hz}$, $1 \mathrm{H}), 7.33-7.14(\mathrm{~m}, 4 \mathrm{H}), 7.10(\mathrm{t}, J=7.9 \mathrm{~Hz}, 1 \mathrm{H}), 5.52(\mathrm{~s}, 1 \mathrm{H})$, $4.11(\mathrm{~s}, 3 \mathrm{H}), 3.74(\mathrm{~s}, 3 \mathrm{H})$.

3-(5-methoxy-1-methyl-1H-indol-3-yl)-2-(1-methyl-1H-indol-3-yl)-3oxopropanenitrile $(\mathbf{1 8 h})$

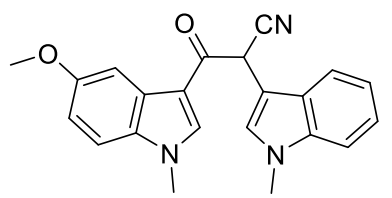

${ }^{1} \mathrm{H}$ NMR (300 MHz, Chloroform- $d$ ) $\delta 7.87$ (d, $J=2.5 \mathrm{~Hz}$, $1 \mathrm{H}), 7.76(\mathrm{~s}, 1 \mathrm{H}), 7.72(\mathrm{dt}, J=7.8,1.0 \mathrm{~Hz}, 1 \mathrm{H}), 7.32-7.12$ (m, 5H), $6.93(\mathrm{dd}, J=8.9,2.5 \mathrm{~Hz}, 1 \mathrm{H}), 5.52(\mathrm{~d}, J=0.7 \mathrm{~Hz}$, $1 \mathrm{H}), 3.86(\mathrm{~s}, 3 \mathrm{H}), 3.72(\mathrm{~s}, 3 \mathrm{H}), 3.71(\mathrm{~s}, 3 \mathrm{H})$.

3-(7-methoxy-1-methyl-1H-indol-3-yl)-2-(1-methyl-1H-indol-3-yl)-3oxopropanenitrile (18i)

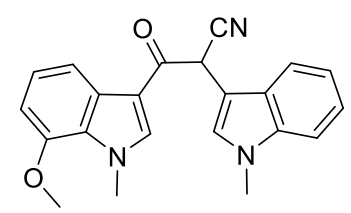

${ }^{1} \mathrm{H}$ NMR (300 MHz, Chloroform- $d$ ) $\delta 7.97$ (d, $\left.J=8.1 \mathrm{~Hz}, 1 \mathrm{H}\right)$, $7.76-7.69(\mathrm{~m}, 1 \mathrm{H}), 7.66(\mathrm{~s}, 1 \mathrm{H}), 7.34-7.24(\mathrm{~m}, 2 \mathrm{H}), 7.24-$ $7.10(\mathrm{~m}, 3 \mathrm{H}), 6.70$ (dd, J = 7.9, $0.9 \mathrm{~Hz}, 1 \mathrm{H}), 5.51$ (s, 1H), 4.00 (s, 3H), 3.89 (s, 3H), 3.72 (s, 3H).

tert-butyl (2-(3-(2-cyano-2-(1-methyl-1H-indol-3-yl)acetyl)-1H-indol-1yl)ethyl)carbamate (18j)<smiles>Cn1cc(C(C(=O)c2cn(CCNC(=O)O)c3ccccc23)c2ccccc2)c2ccccc21</smiles>

${ }^{1} \mathrm{H}$ NMR (300 MHz, Chloroform- $d$ ) $\delta 8.40-8.30$ (m, 1H), 7.88 (s, 1H), $7.71(\mathrm{~d}, J=7.9 \mathrm{~Hz}, 1 \mathrm{H}), 7.40-7.13(\mathrm{~m}, 8 \mathrm{H}), 5.52(\mathrm{~d}, J$ $=0.8 \mathrm{~Hz}, 1 \mathrm{H}), 4.75(\mathrm{~s}, 1 \mathrm{H}), 4.24(\mathrm{~s}, 2 \mathrm{H}), 3.73(\mathrm{~s}, 3 \mathrm{H}), 3.47(\mathrm{~d}, J$ $=6.0 \mathrm{~Hz}, 2 \mathrm{H}), 1.41(\mathrm{~s}, 9 \mathrm{H})$. 
tert-butyl 4-(3-(2-cyano-2-(1-methyl-1H-indol-3-yl)acetyl)-1H-indol-1-yl)piperidine1-carboxylate (181)

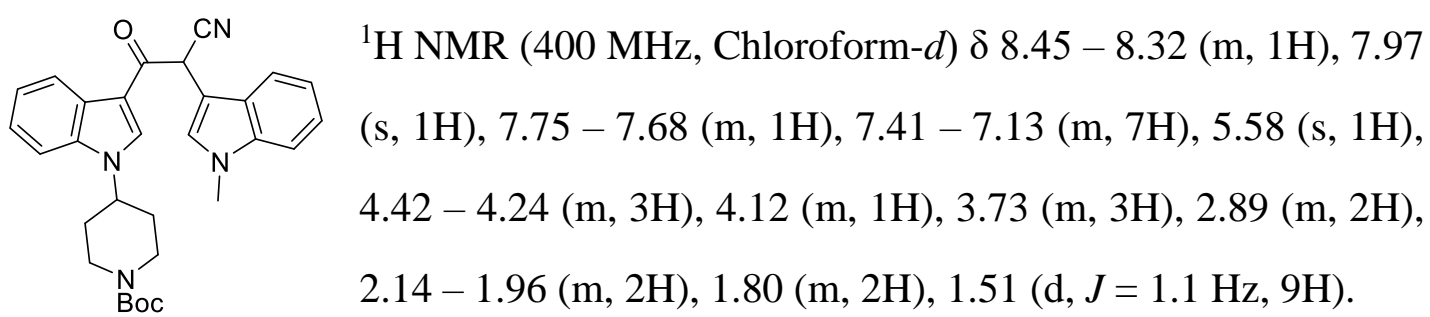

2-(1-methyl-1H-indol-3-yl)-3-oxo-3-(2-(piperidine-1-carbonyl)-1,2,3,4-tetrahydro[1,4]diazepino[6,7,1-hi]indol-7-yl)propanenitrile (25a)

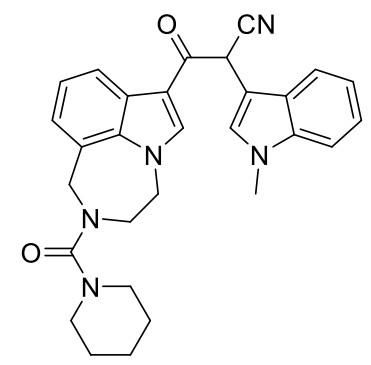

${ }^{1} \mathrm{H}$ NMR (300 MHz, Chloroform- $\left.d\right) \delta 8.37-8.25(\mathrm{~m}, 1 \mathrm{H})$,

$7.83(\mathrm{~s}, 1 \mathrm{H}), 7.72(\mathrm{~d}, J=7.9 \mathrm{~Hz}, 1 \mathrm{H}), 7.34-7.01(\mathrm{~m}, 6 \mathrm{H})$,

$5.55(\mathrm{~s}, 1 \mathrm{H}), 4.64(\mathrm{~s}, 2 \mathrm{H}), 4.26(\mathrm{q}, J=3.5 \mathrm{~Hz}, 2 \mathrm{H}), 3.91(\mathrm{~d}, J$

$=5.1 \mathrm{~Hz}, 2 \mathrm{H}), 3.70(\mathrm{~s}, 3 \mathrm{H}), 3.12(\mathrm{~m}, 4 \mathrm{H}), 1.55(\mathrm{~s}, 6 \mathrm{H})$.

tert-butyl 7-(2-cyano-2-(1-methyl-1H-indol-3-yl)acetyl)-3,4-

dihydro-[1,4]diazepino[6,7,1-hi]indole-2(1H)-carboxylate $\mathbf{( 2 5 b )}$

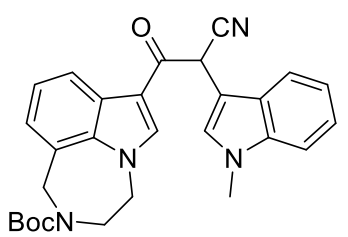

${ }^{1} \mathrm{H}$ NMR (400 MHz, Chloroform- $d$ ) $\delta 8.31(\mathrm{~d}, J=8.0 \mathrm{~Hz}$,

$1 \mathrm{H}), 7.82(\mathrm{~d}, J=3.4 \mathrm{~Hz}, 1 \mathrm{H}), 7.71(\mathrm{~d}, J=8.0 \mathrm{~Hz}, 1 \mathrm{H}), 7.35$

$-7.01(\mathrm{~m}, 6 \mathrm{H}), 5.53(\mathrm{~s}, 1 \mathrm{H}), 4.84(\mathrm{~d}, J=36.3 \mathrm{~Hz}, 2 \mathrm{H}), 4.34$

(s, 2H), $3.99-3.86(\mathrm{~m}, 2 \mathrm{H}), 3.76(\mathrm{~s}, 3 \mathrm{H}), 1.41(\mathrm{~d}, J=20.1$

$\mathrm{Hz}, 9 \mathrm{H})$. 
2. Copies of NMR spectra of aminopyrazoles 4a-d, 5a-d, 6a-m and 7a-c

4-(1-methyl-1H-indol-3-yl)-5-phenyl-1H-pyrazol-3-amine (4a).
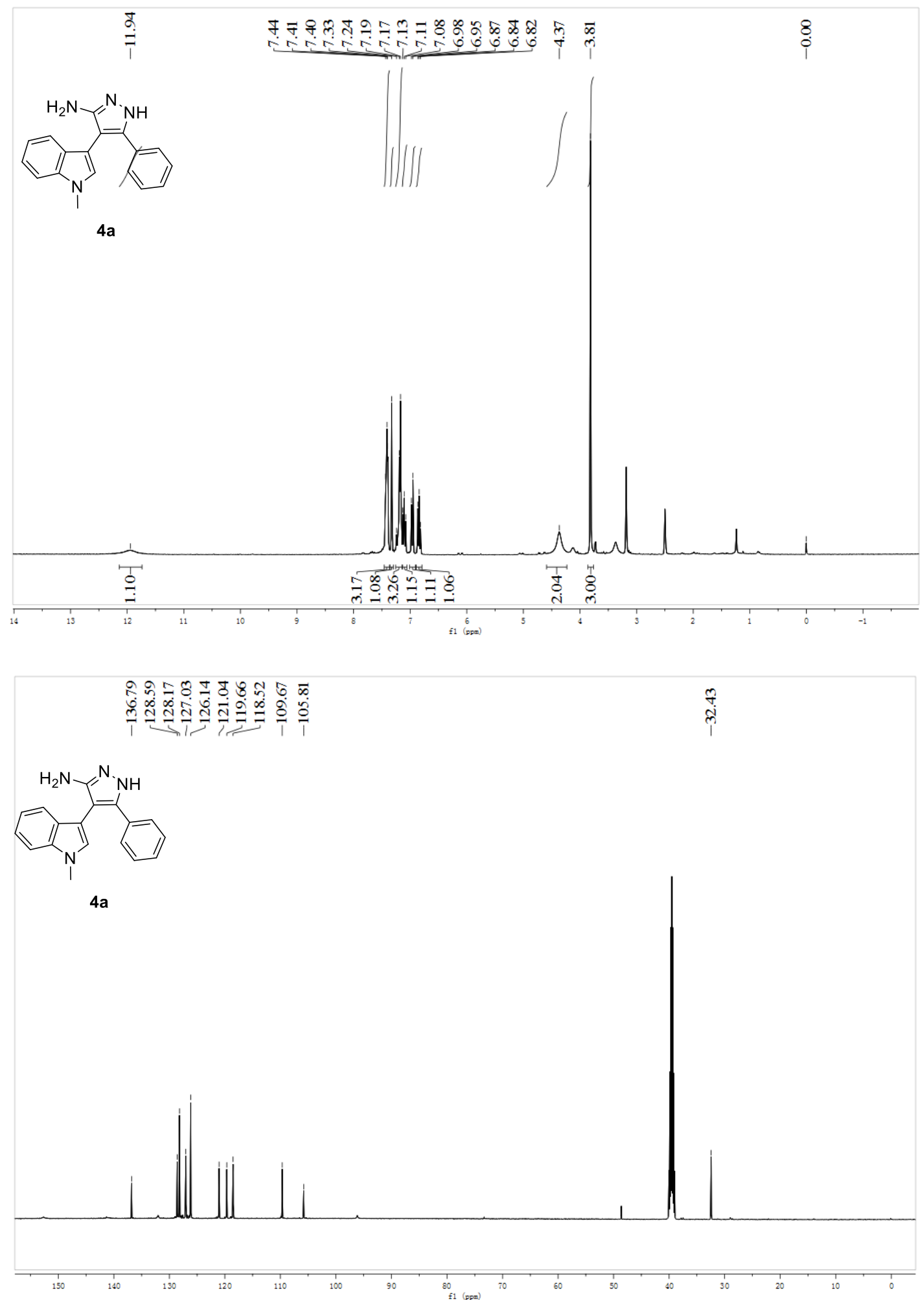
4-(1-methyl-1H-indol-3-yl)-5-(p-tolyl)-1H-pyrazol-3-amine (4b).
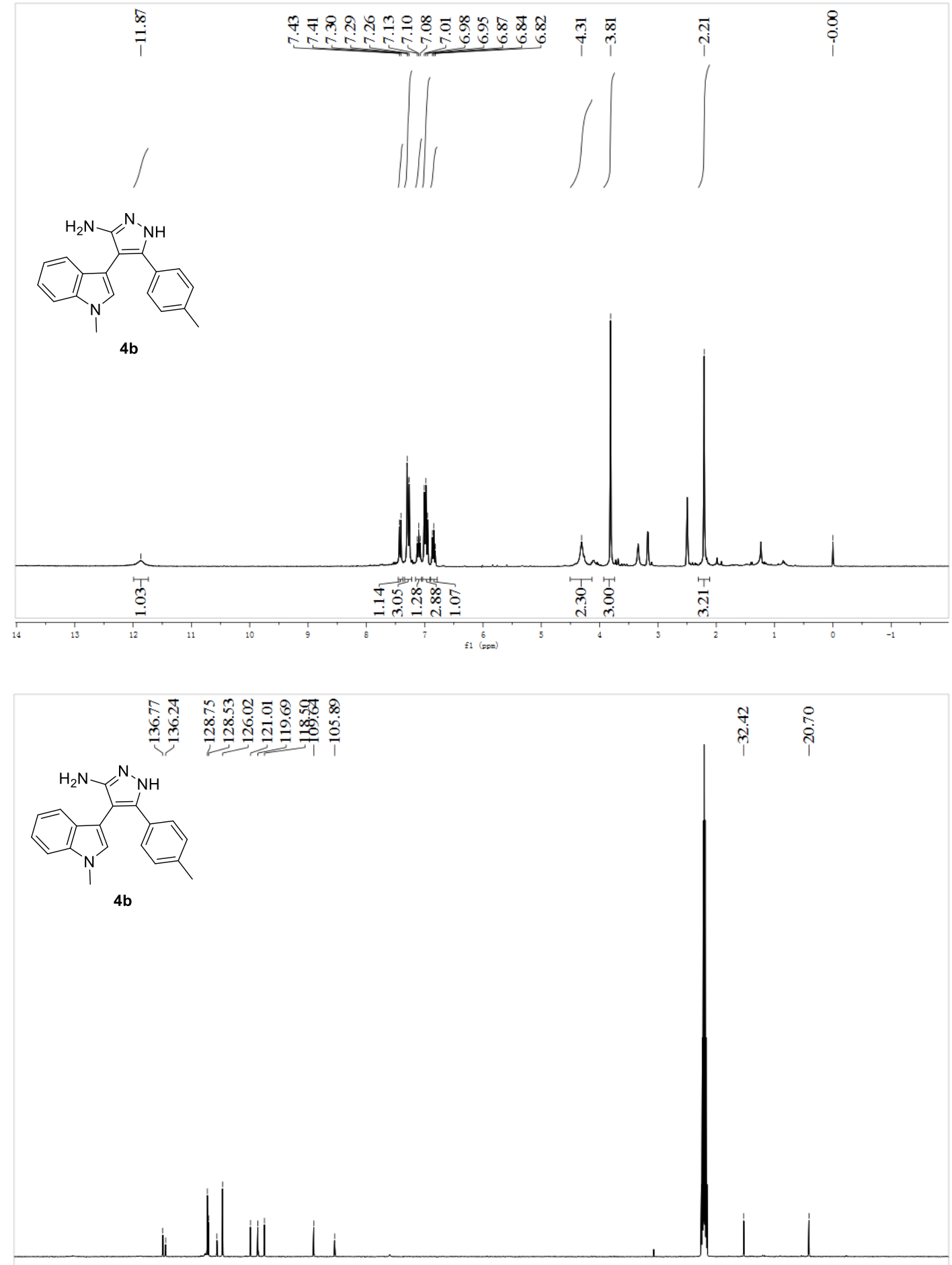

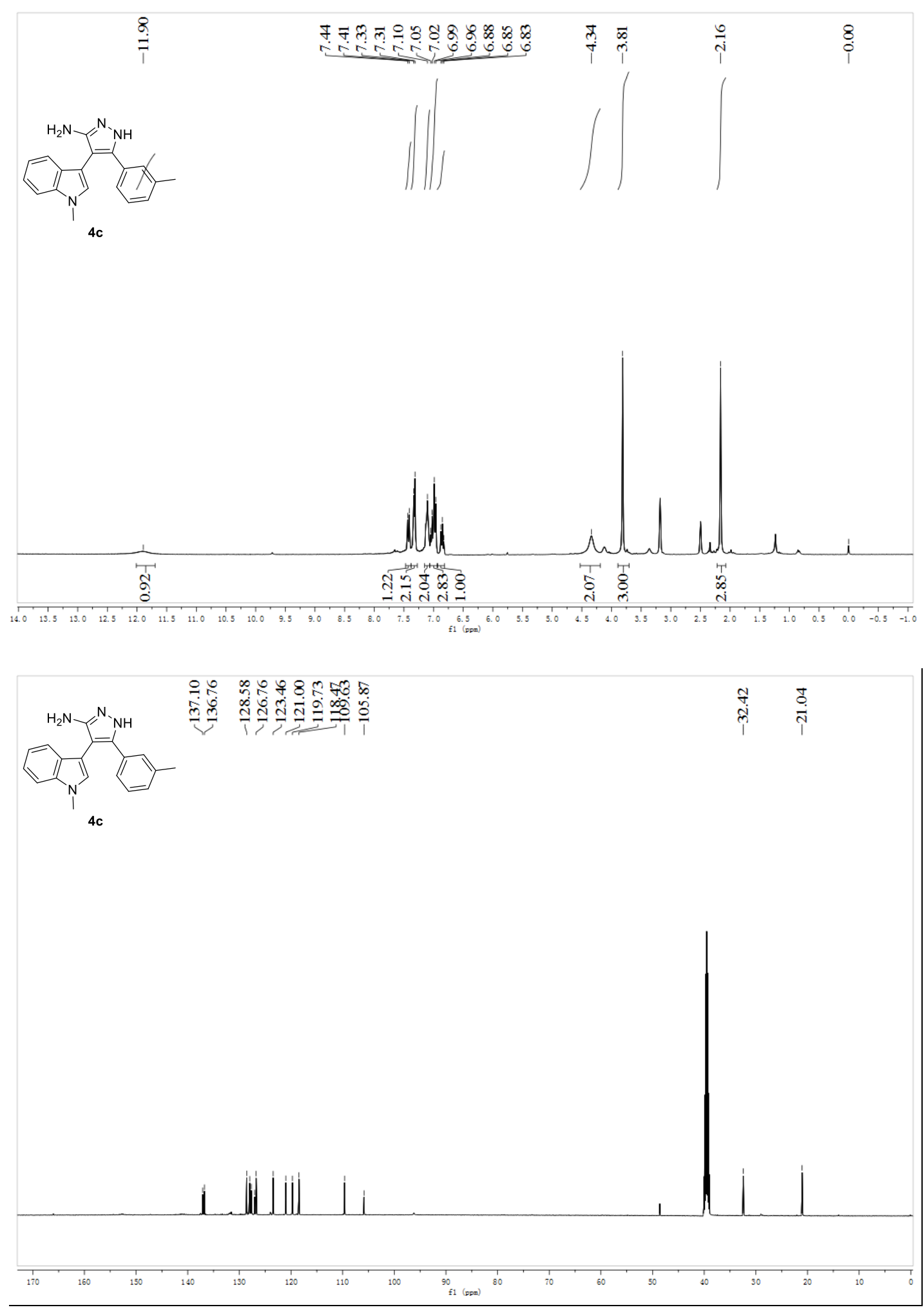
5-(3-bromophenyl)-4-(1-methyl-1H-indol-3-yl)-1H-pyrazol-3-amine (4d).
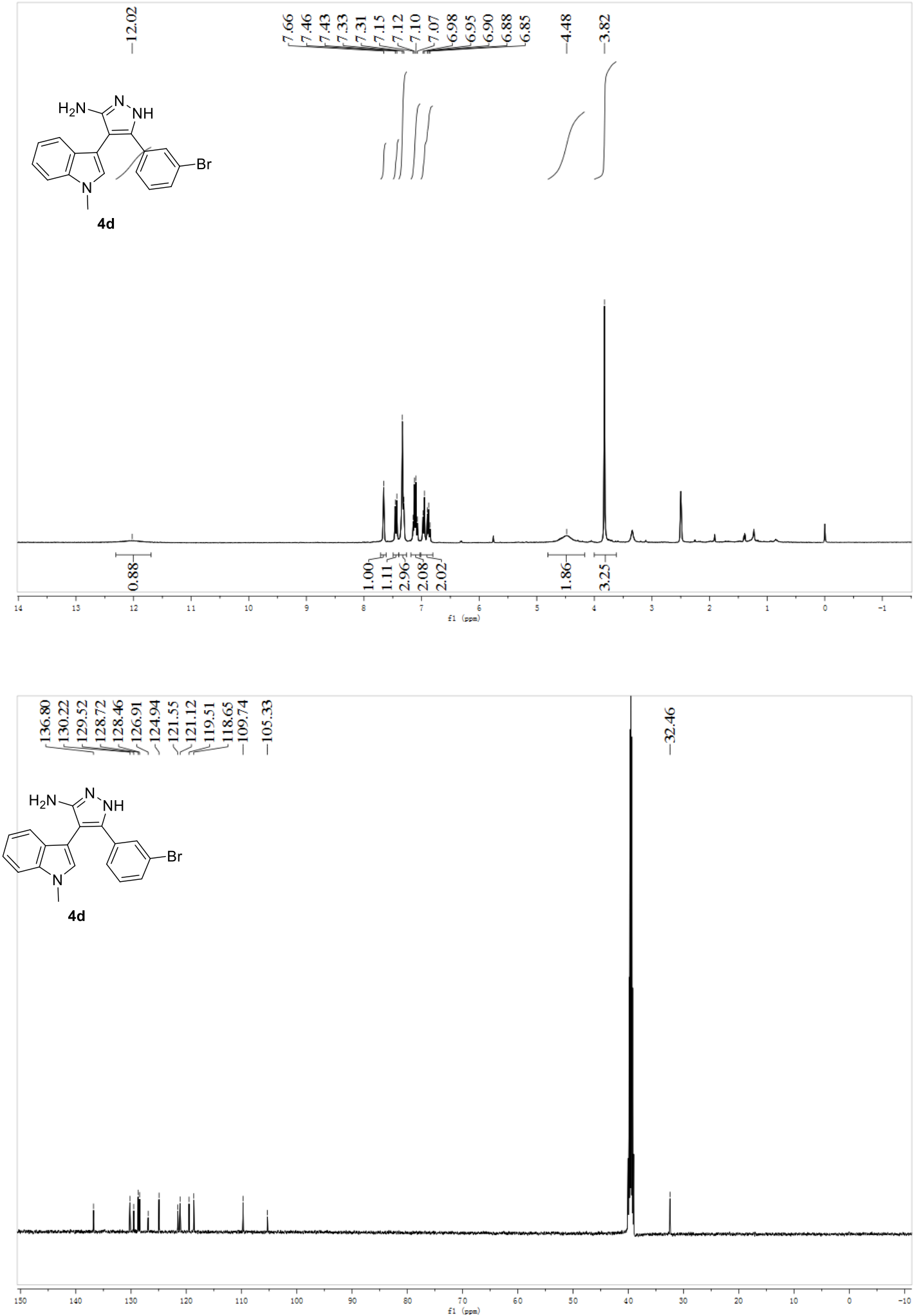
5-(1-methyl-1H-indol-3-yl)-4-phenyl-1H-pyrazol-3-amine (5a).
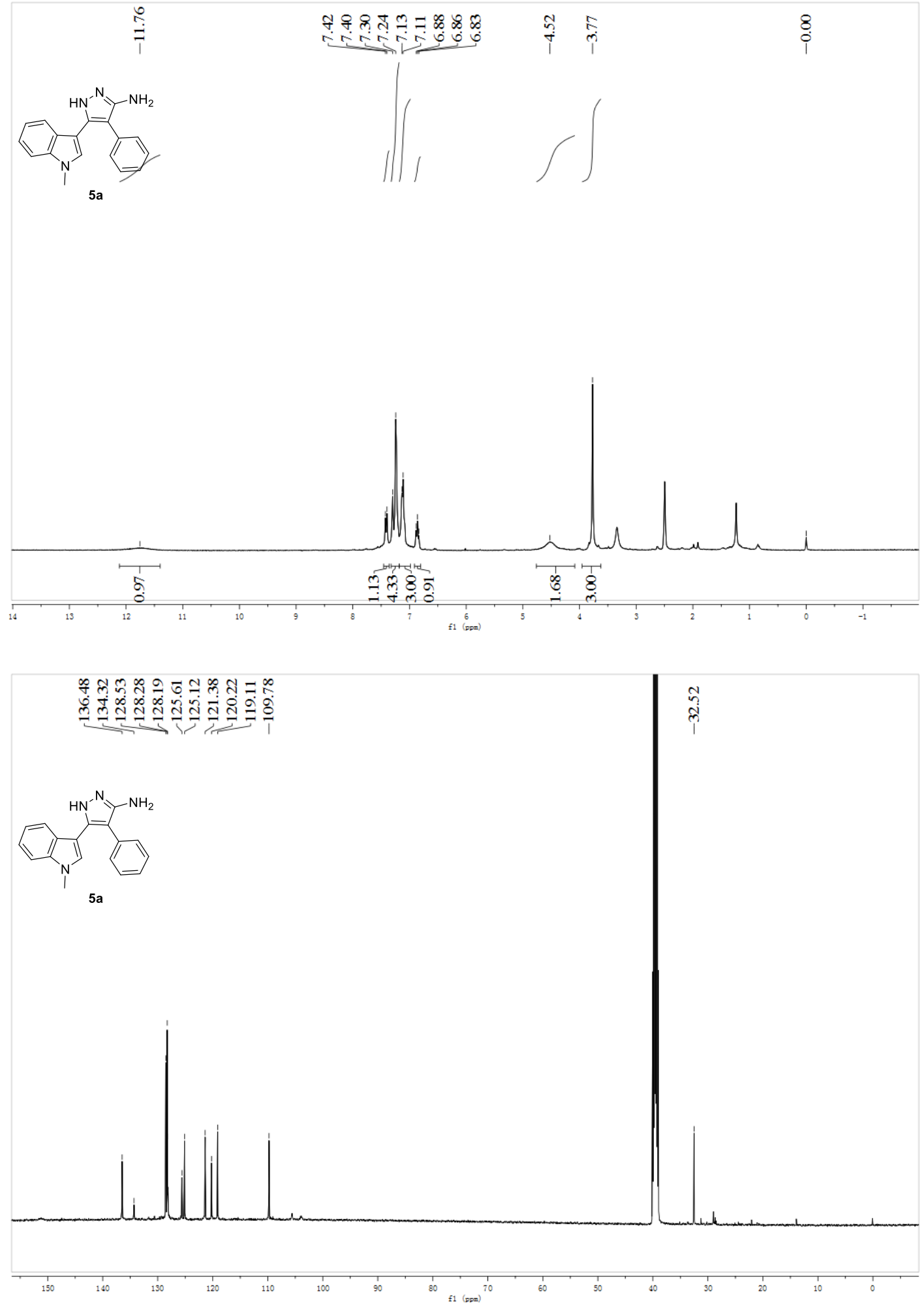
5-(5-methoxy-1-methyl-1H-indol-3-yl)-4-phenyl-1H-pyrazol-3-amine (5b).
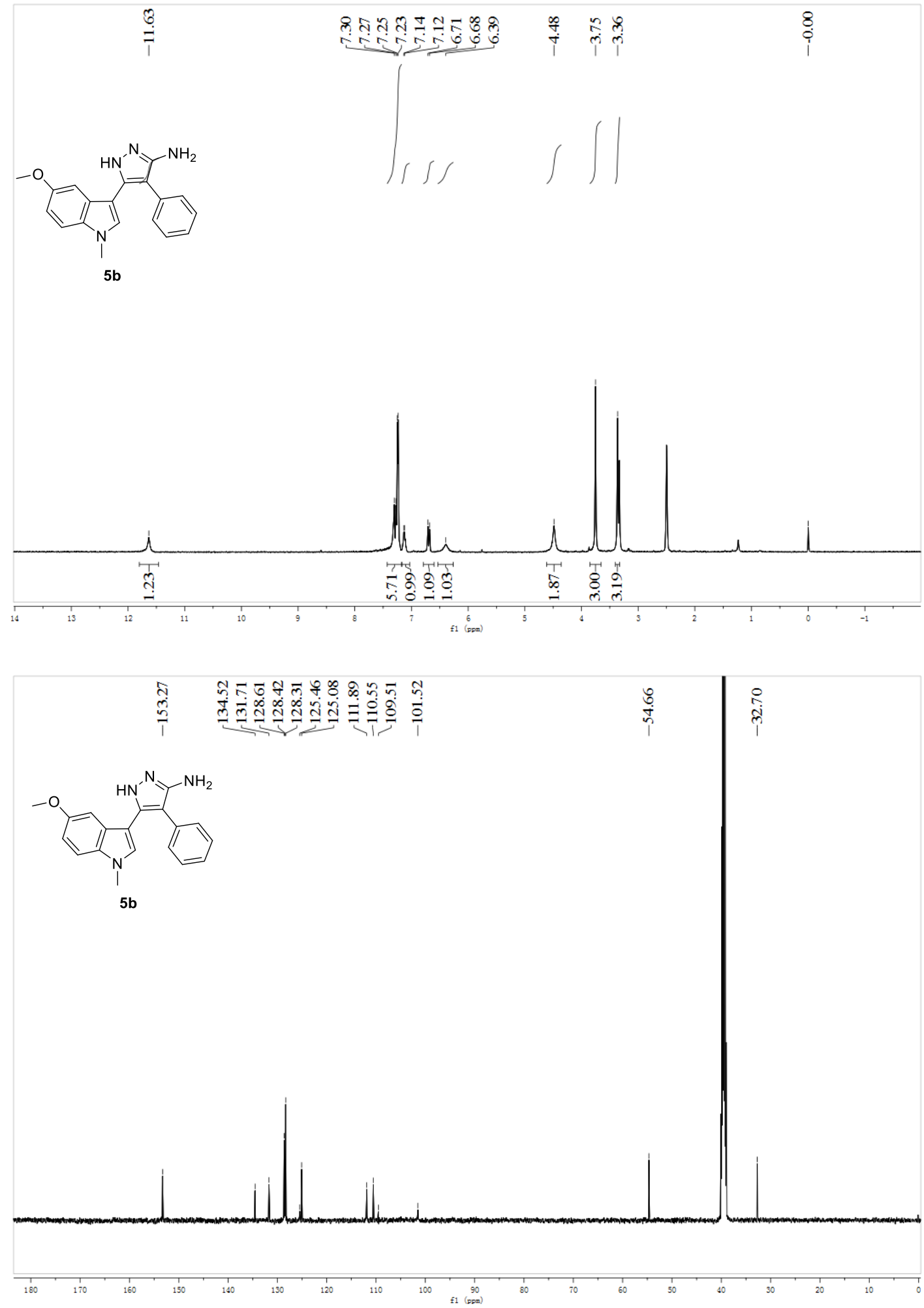
5-(5-bromo-1-methyl-1H-indol-3-yl)-4-phenyl-1H-pyrazol-3-amine (5c).
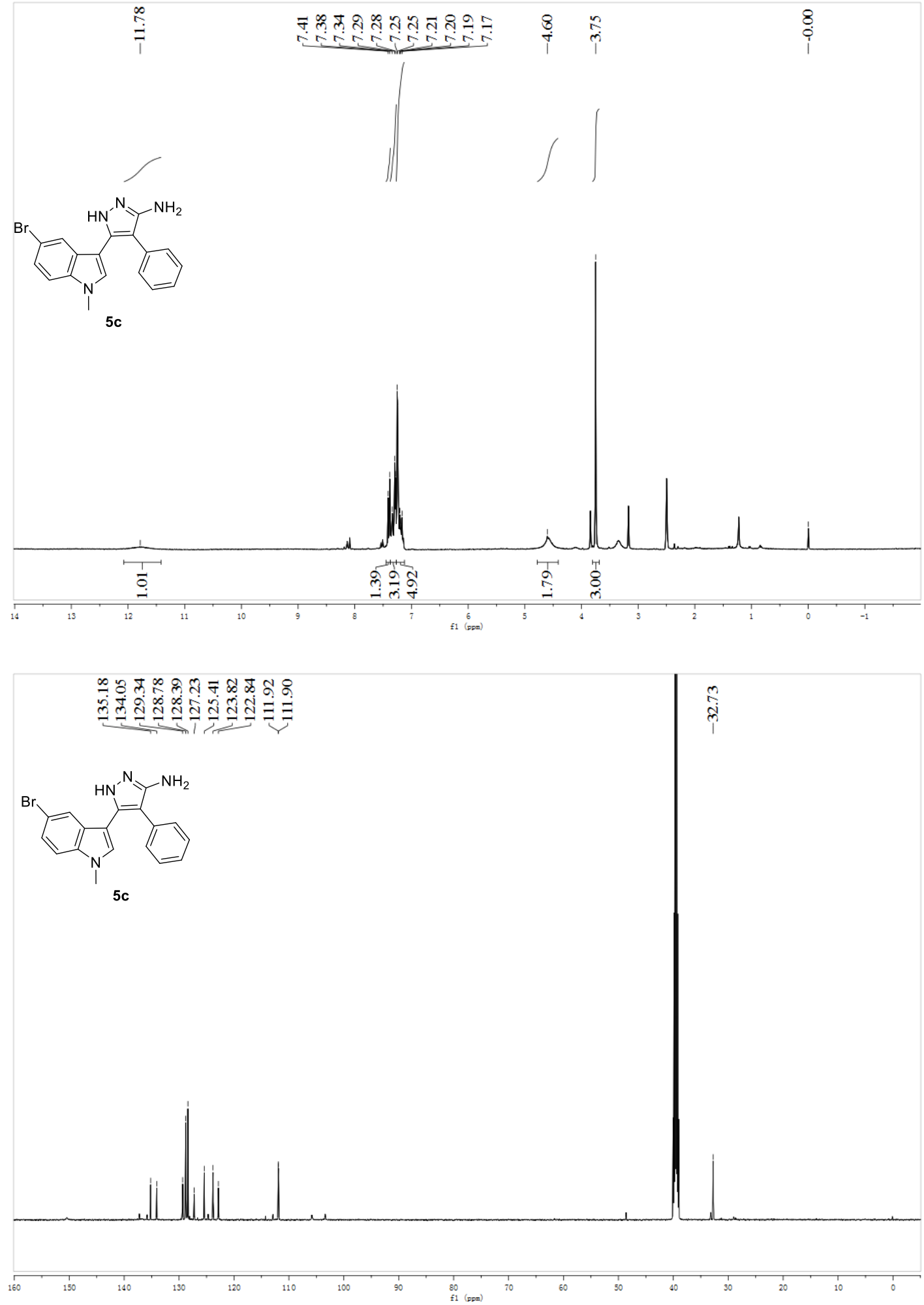
5-(1-methyl-1H-indol-3-yl)-4-(o-tolyl)-1H-pyrazol-3-amine (5d).
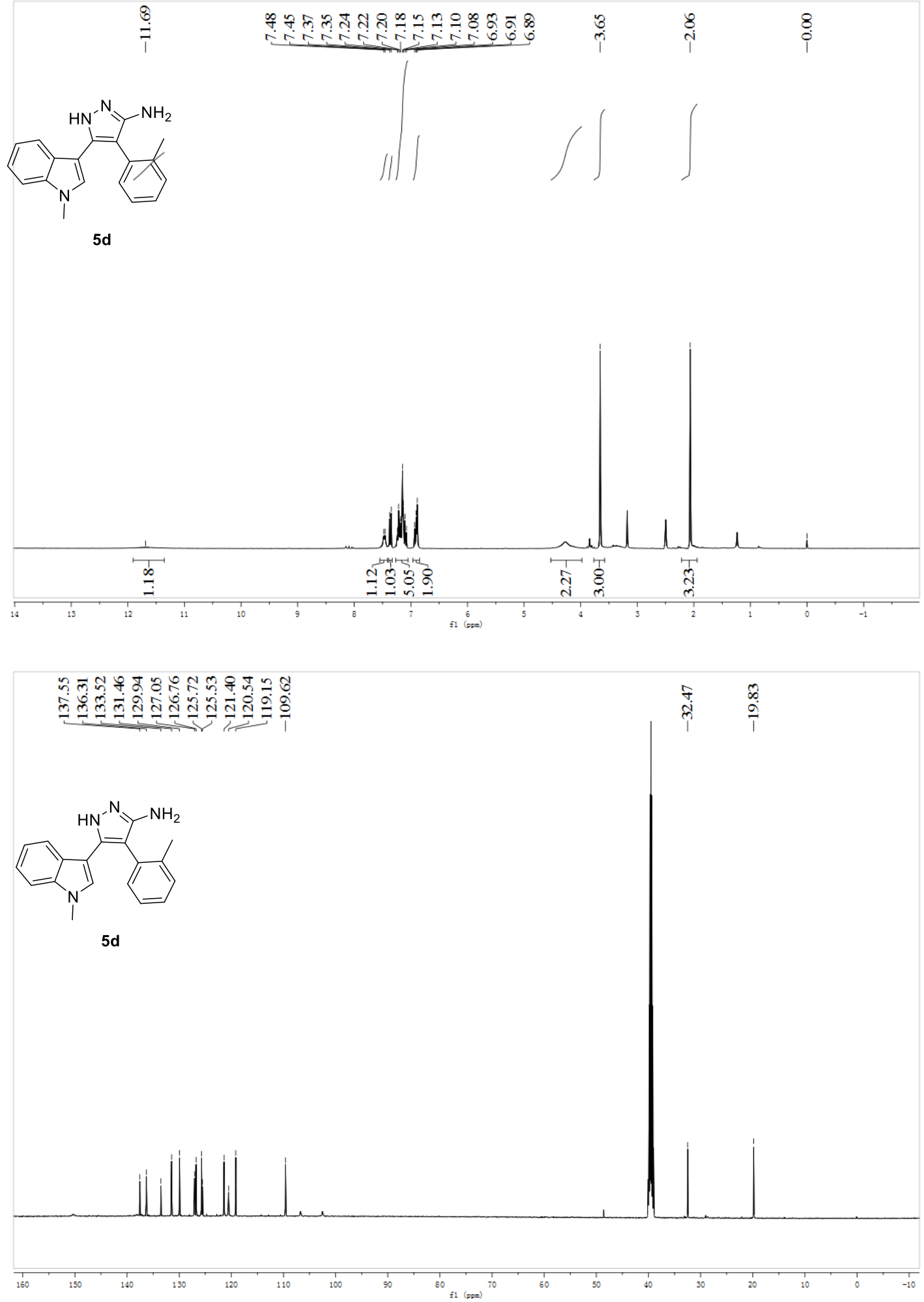
4,5-bis(1-methyl-1H-indol-3-yl)-1H-pyrazol-3-amine (6a).

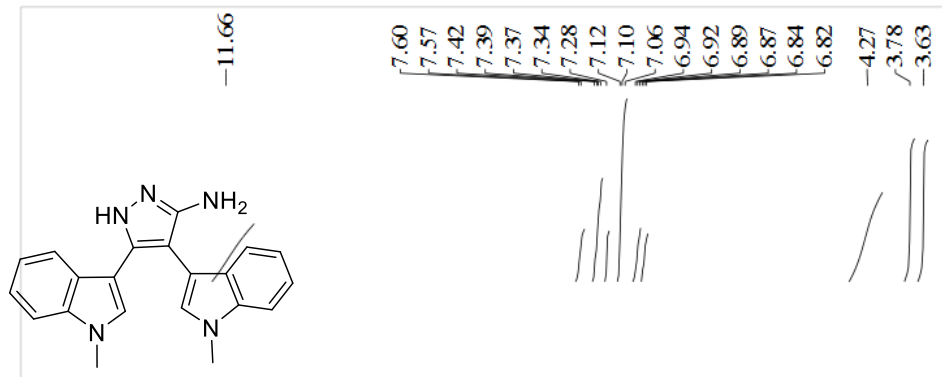

$6 a$

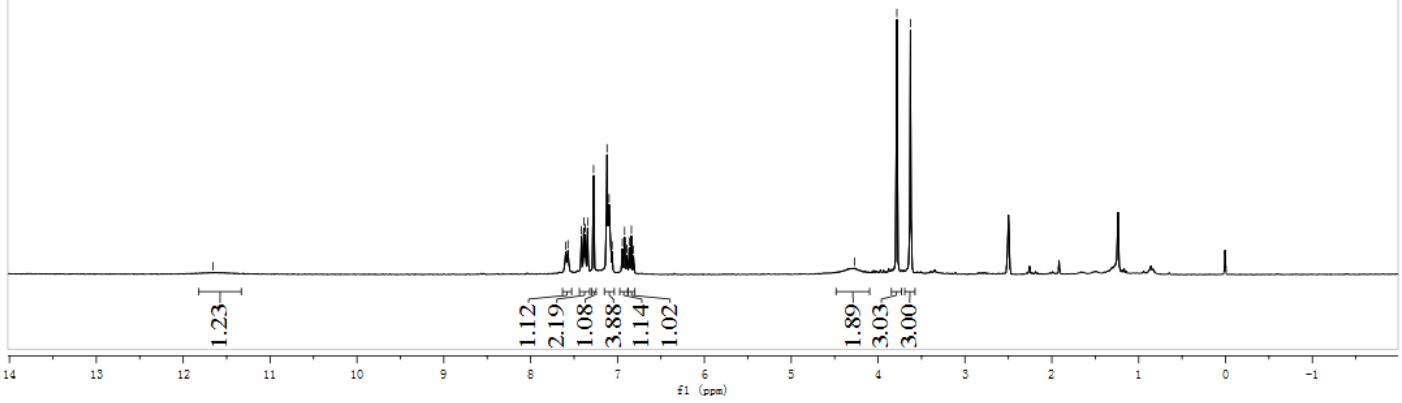

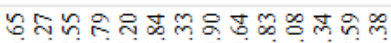

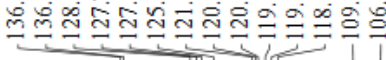

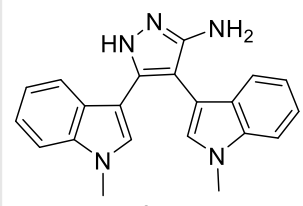

$6 a$

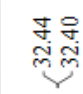
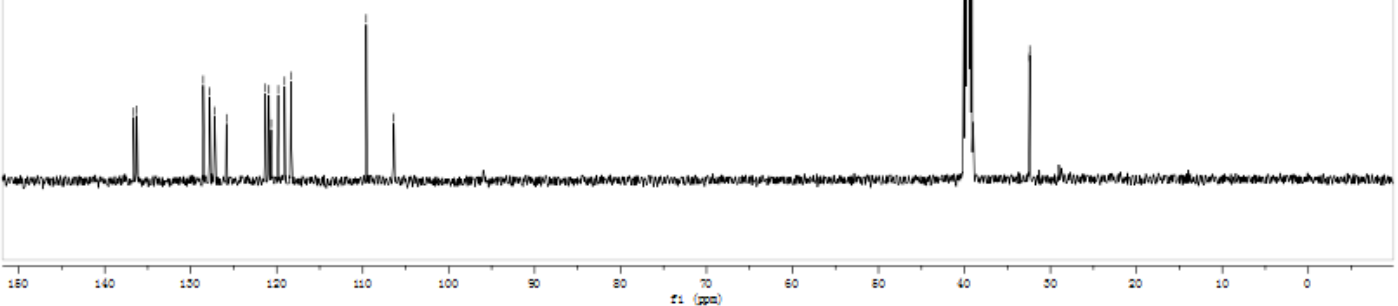
$(6 \mathbf{b})$.
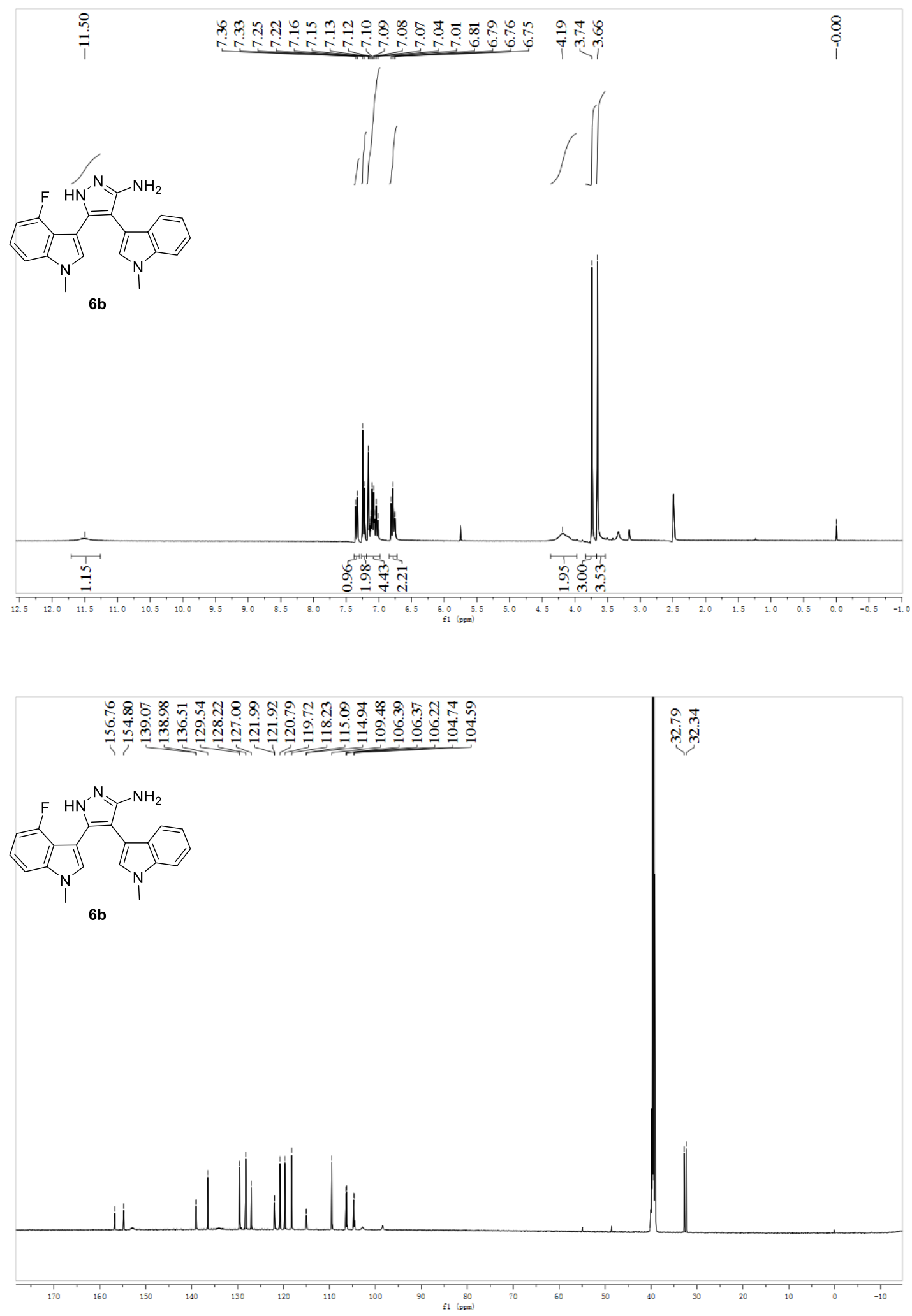

(6c).
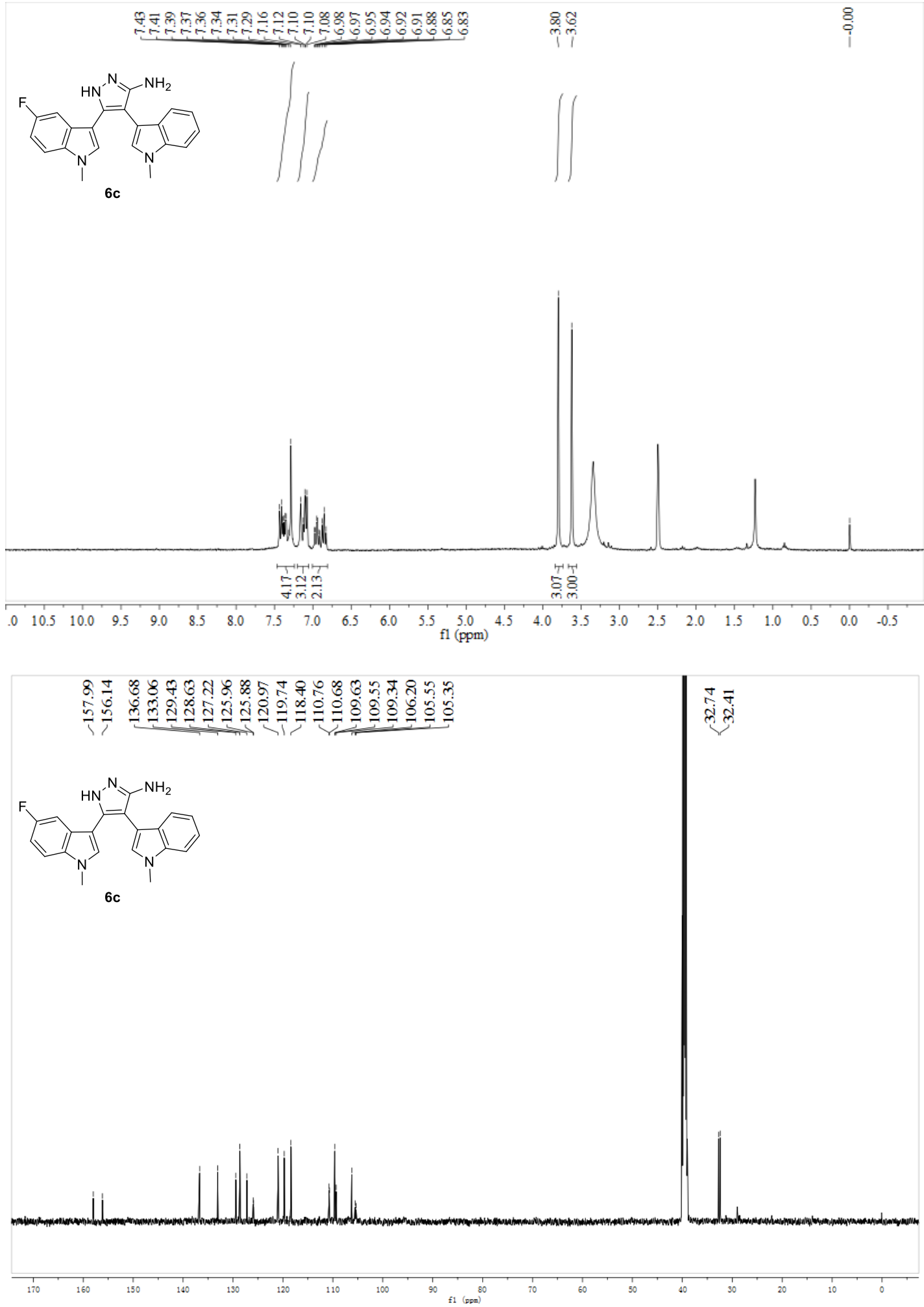

(6d).
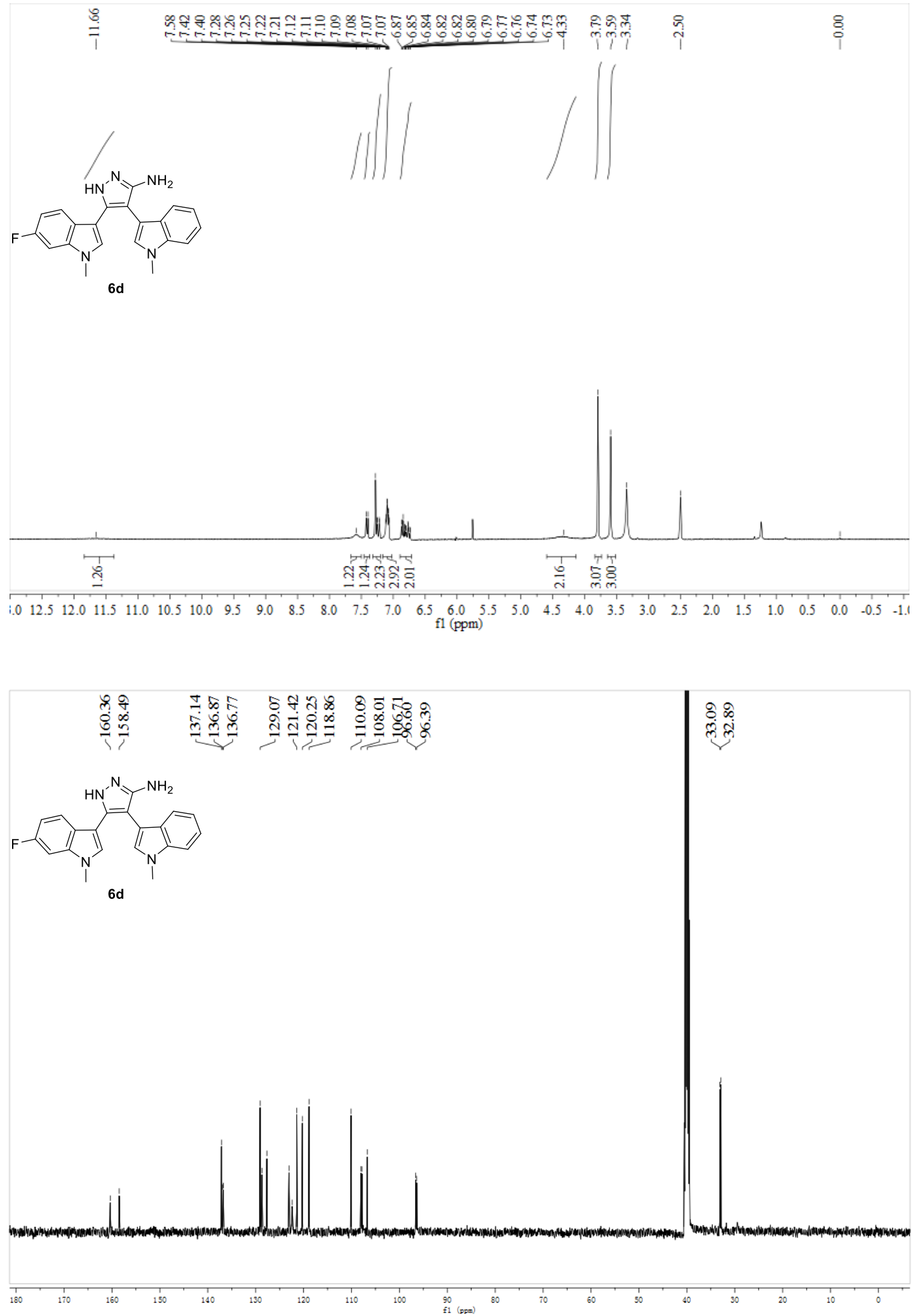

$(6 \mathbf{e})$.
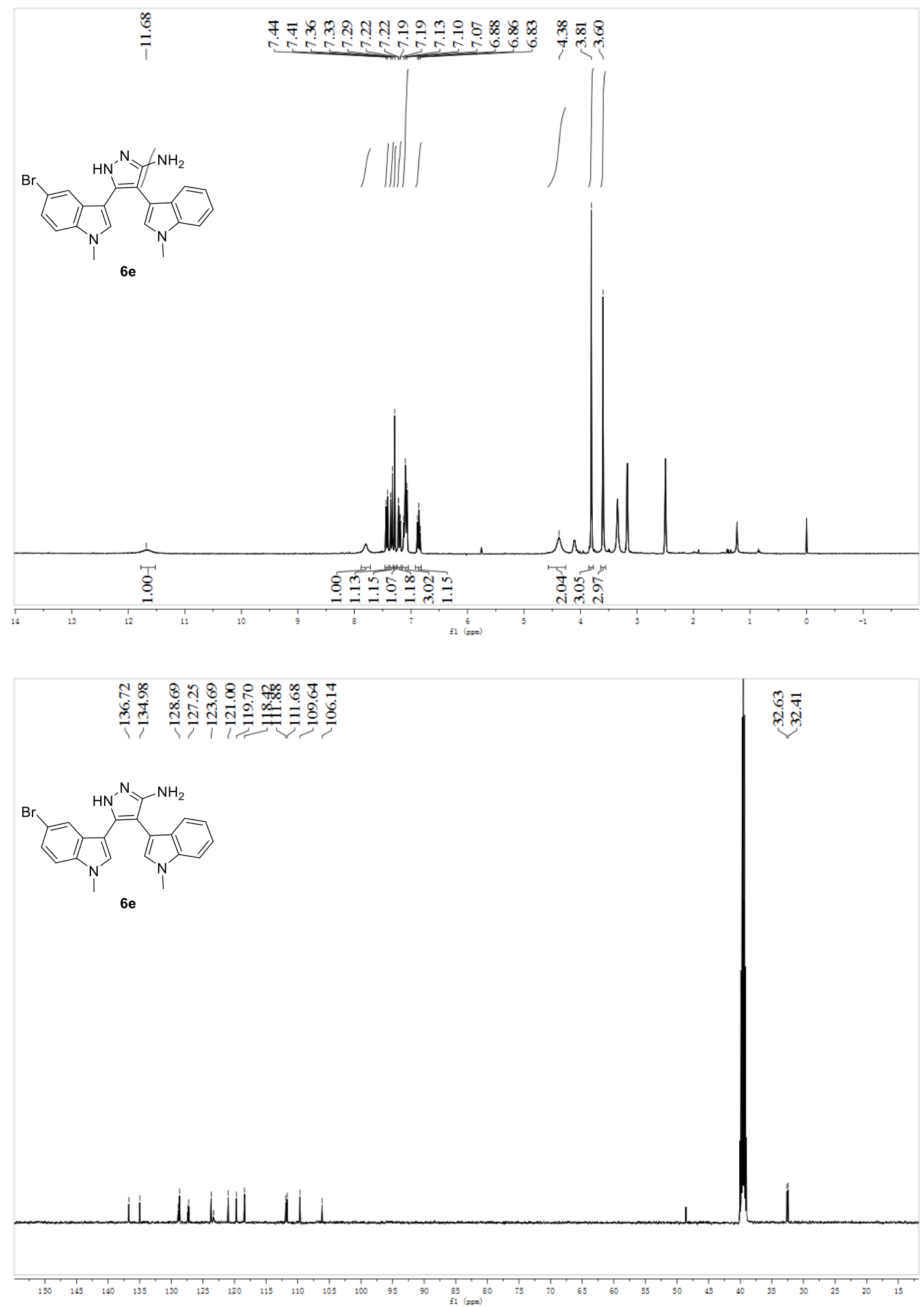

(6f).
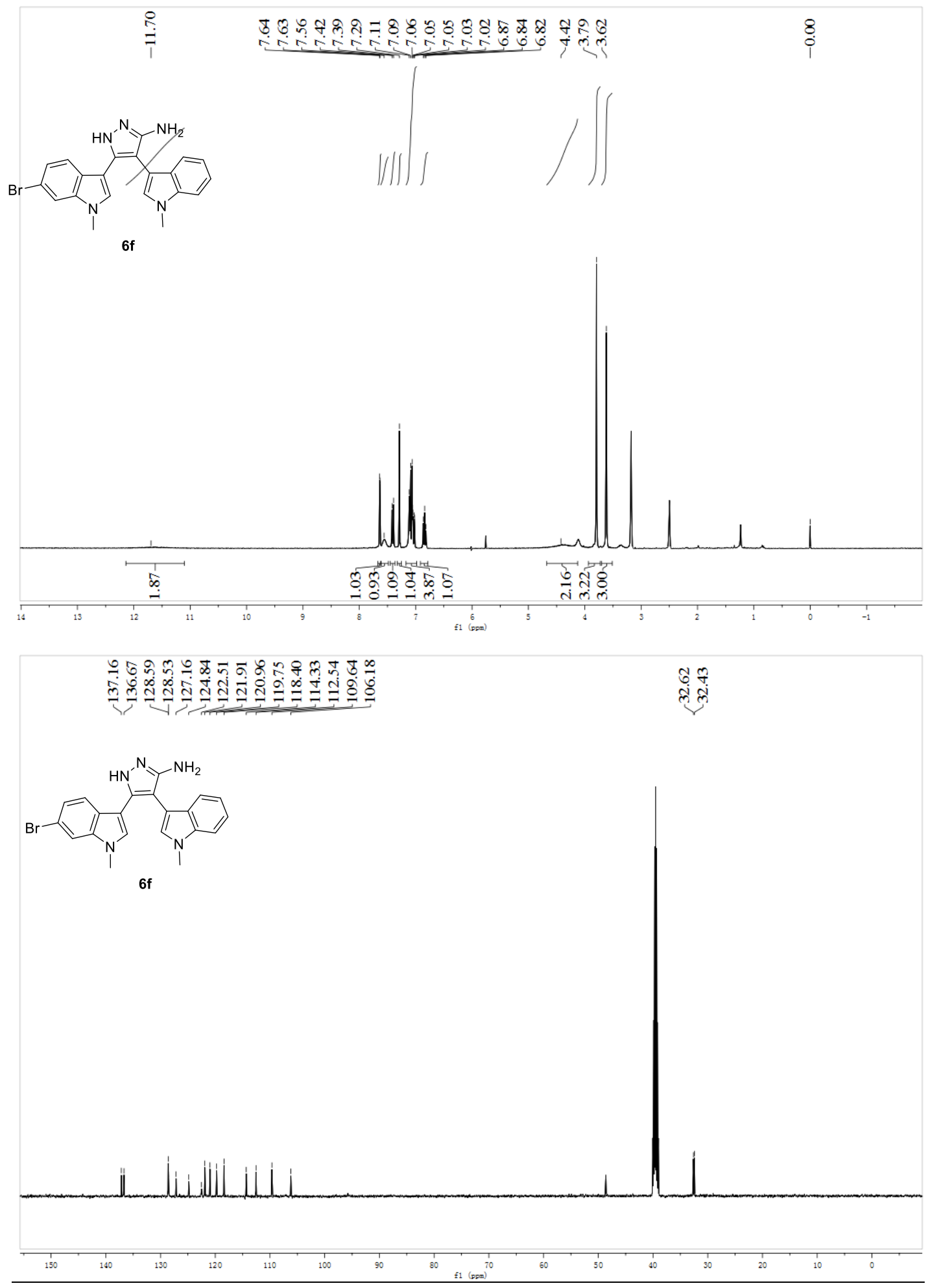
$(6 \mathrm{~g})$

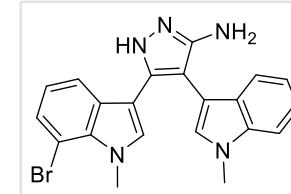

$6 \mathrm{~g}$
6.

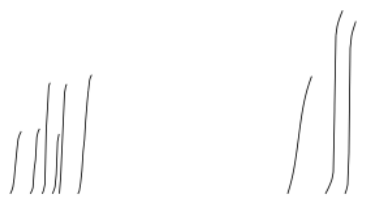

$\stackrel{8}{i}$
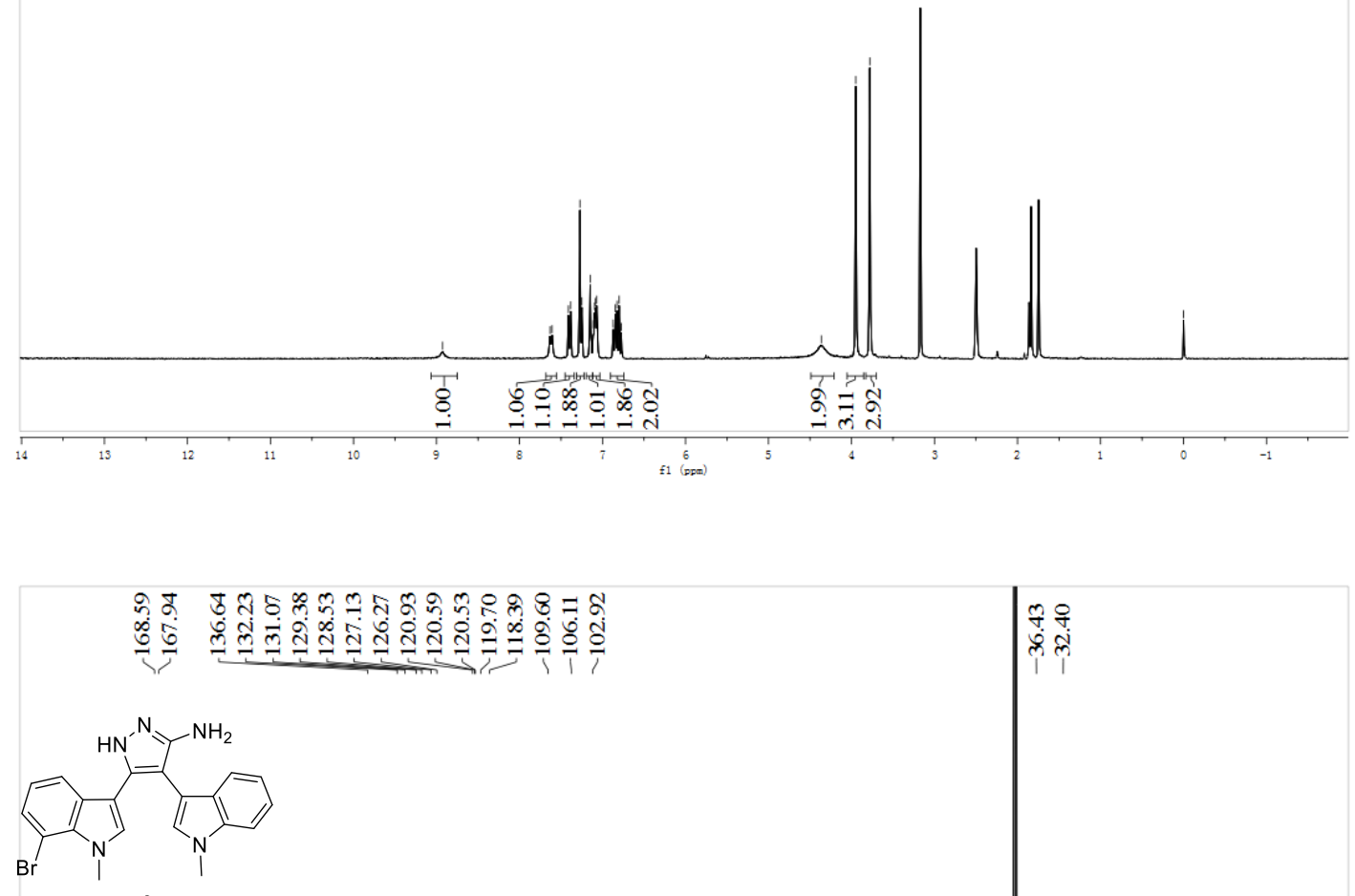

$6 \mathrm{~g}$

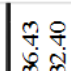
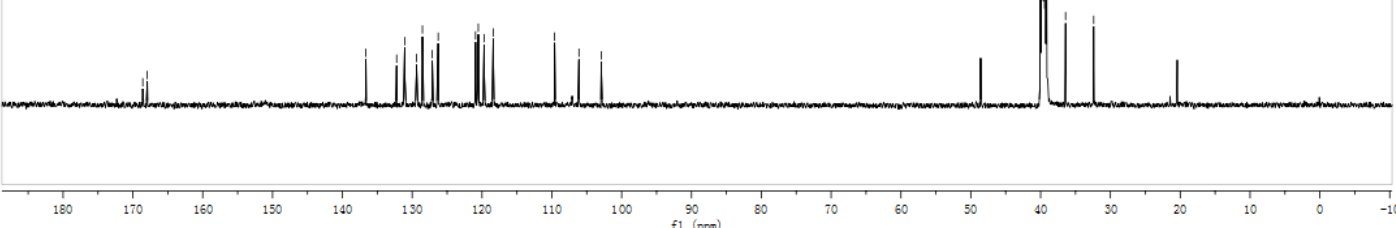
5-(5-methoxy-1-methyl-1H-indol-3-yl)-4-(1-methyl-1H-indol-3-yl)-1H-pyrazol-3amine (6h).
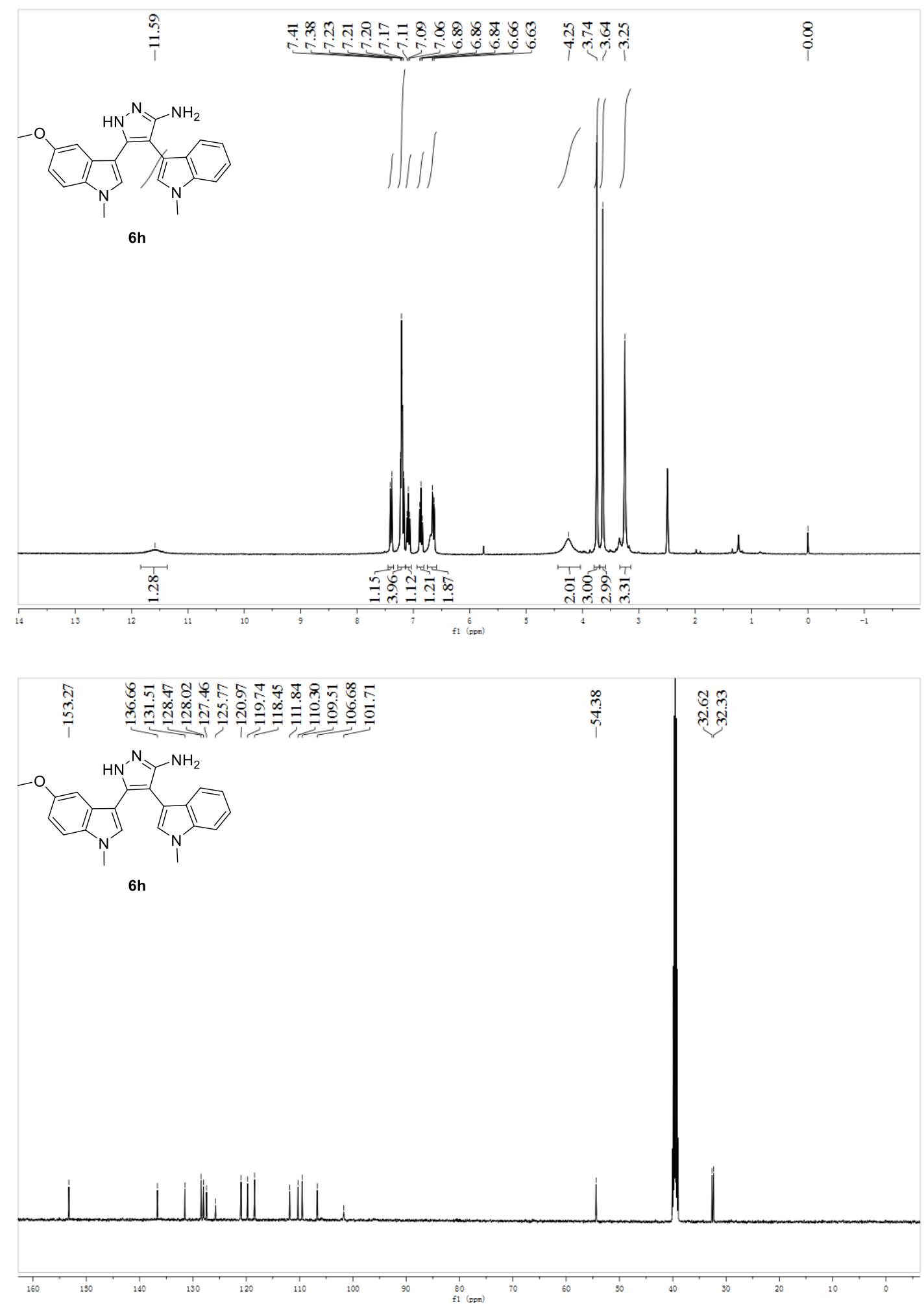
5-(7-methoxy-1-methyl-1H-indol-3-yl)-4-(1-methyl-1H-indol-3-yl)-1H-pyrazol-3amine (6i).
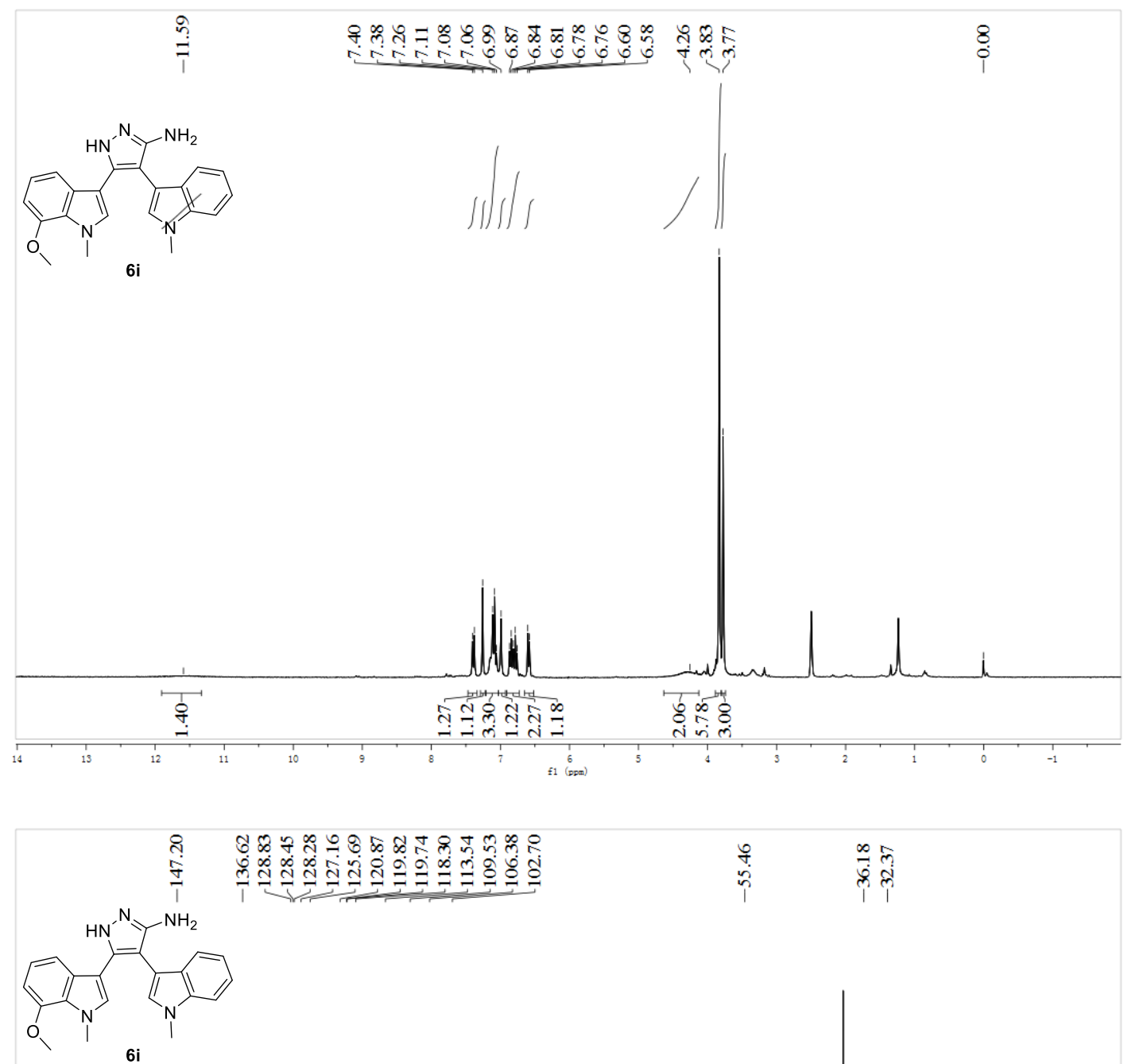

ले

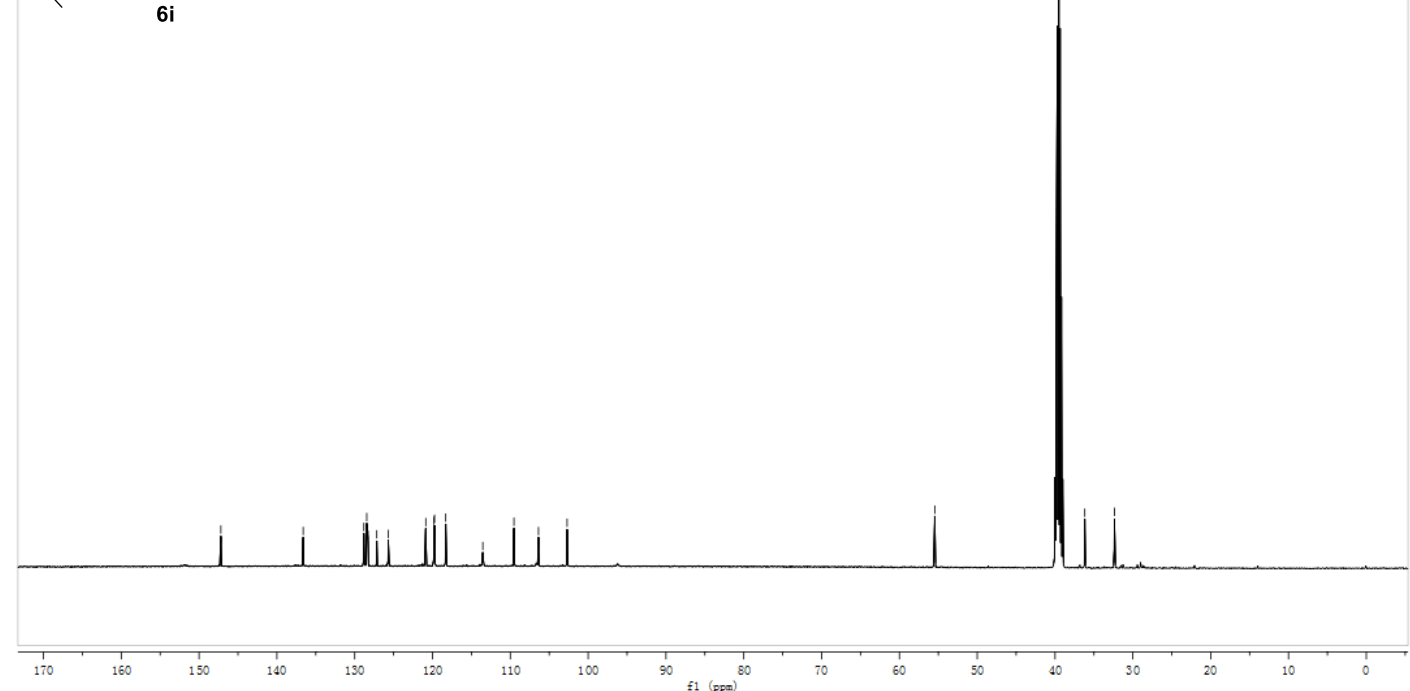


(2-(3-(3-amino-4-(1-methyl-1H-indol-3-yl)-1H-pyrazol-5-yl)-1H-indol-1-

yl)ethyl)carbamate(6j).

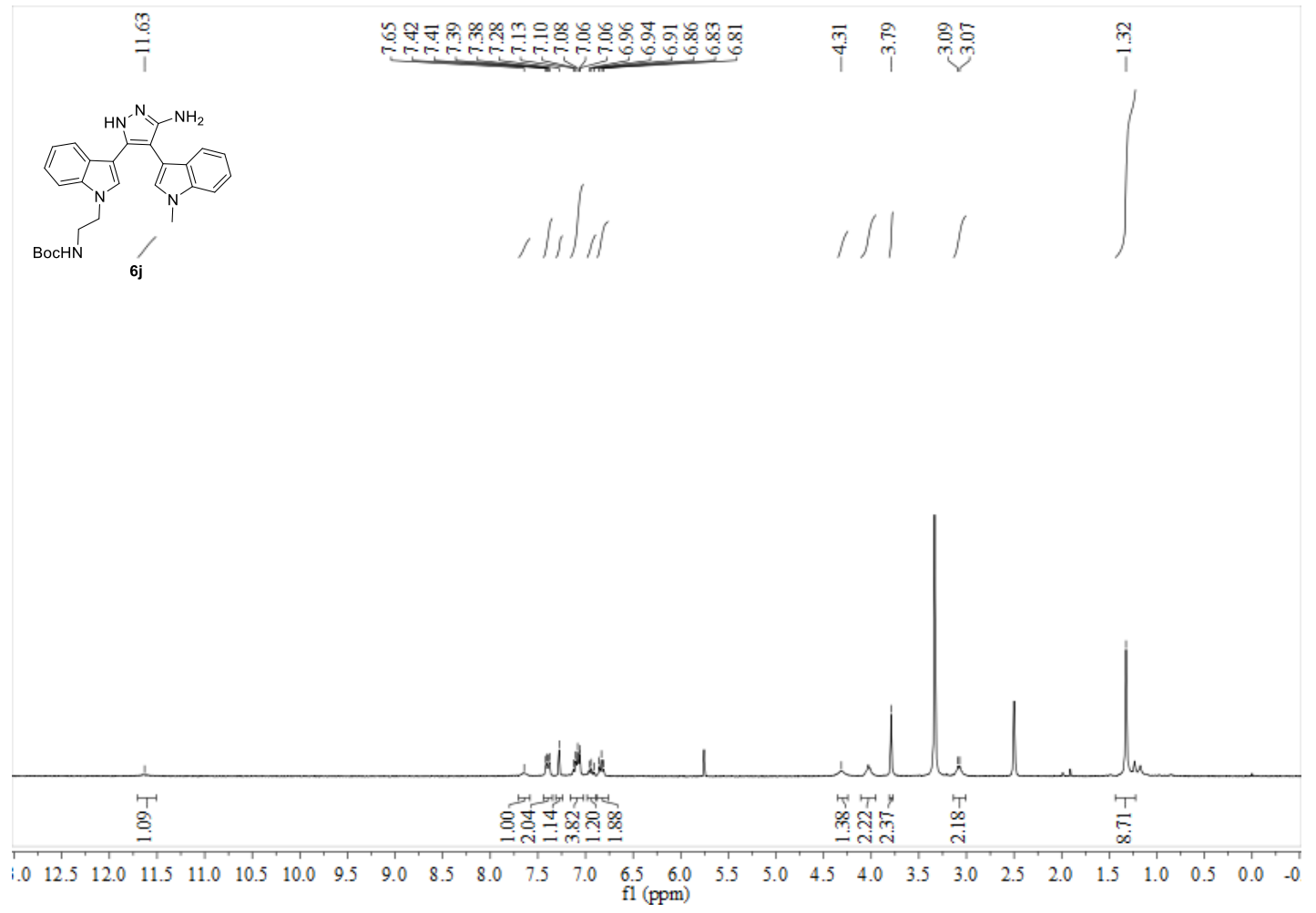

5-(1-(2-aminoethyl)-1H-indol-3-yl)-4-(1-methyl-1H-indol-3-yl)-1H-pyrazol-3-amine $(6 \mathbf{k})$

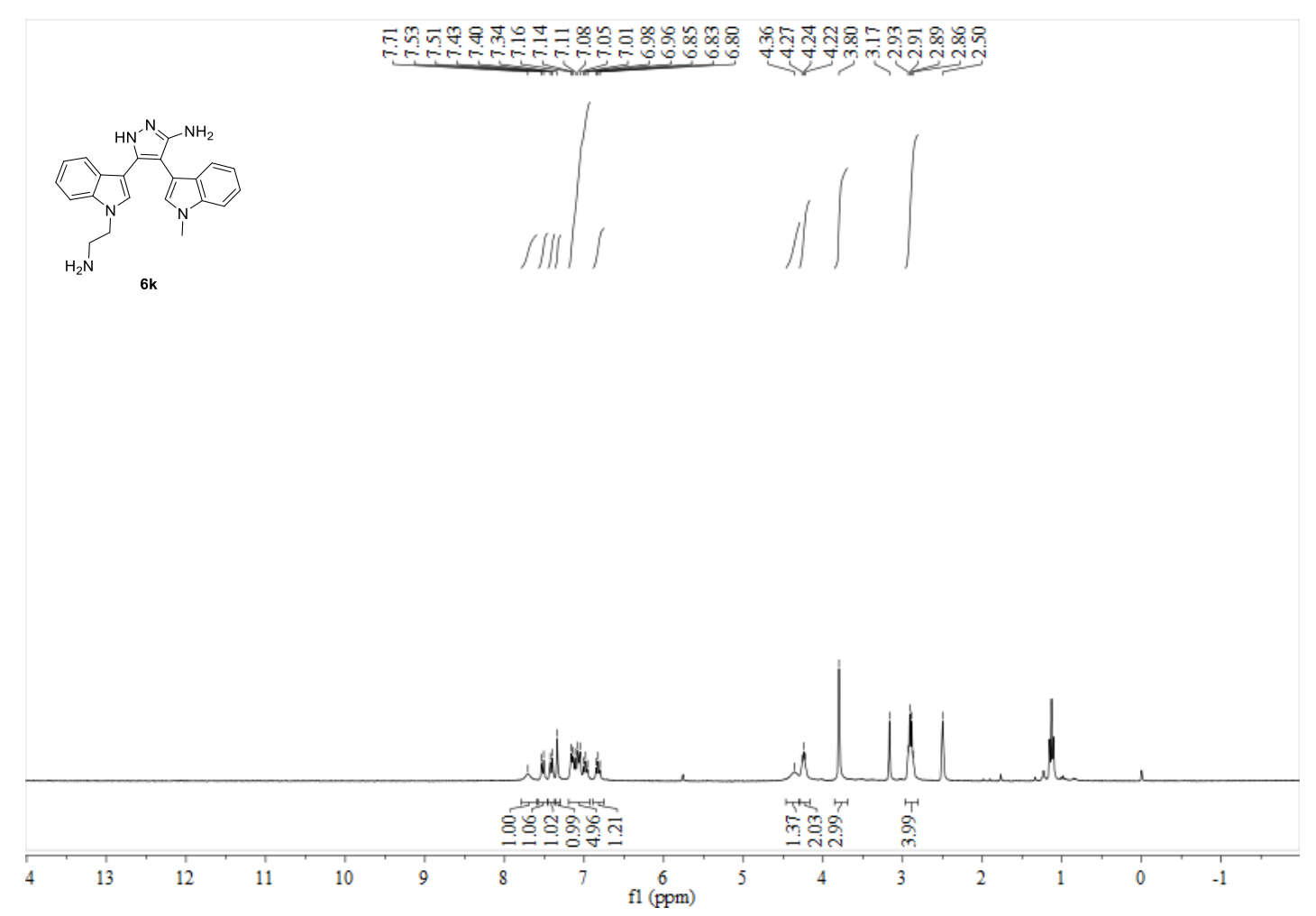


tert-butyl 4-(3-(3-amino-4-(1-methyl-1H-indol-3-yl)-1H-pyrazol-5-yl)-1H-indol-1-

yl)piperidine-1-carboxylate (61).
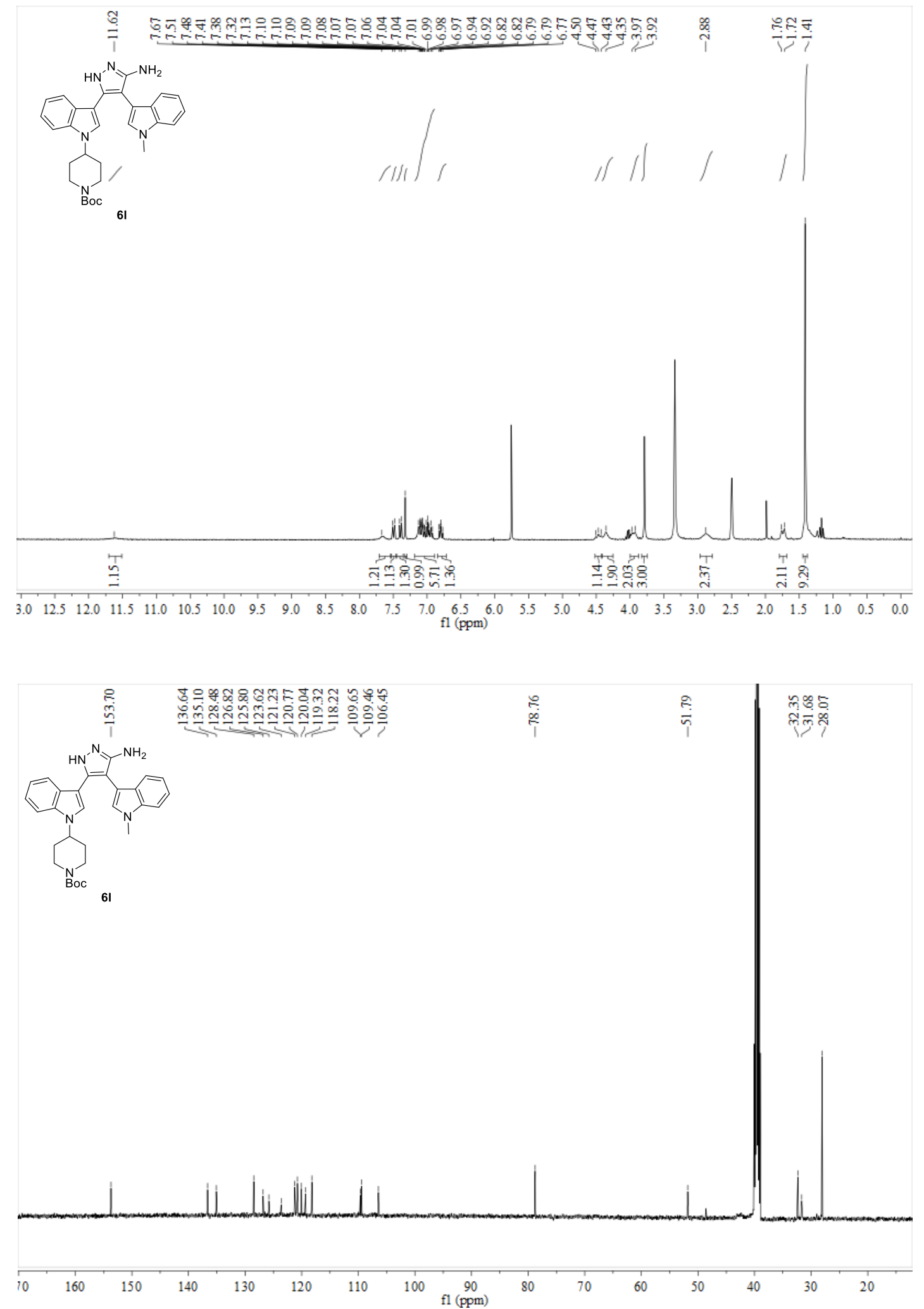

$(6 \mathbf{m})$.

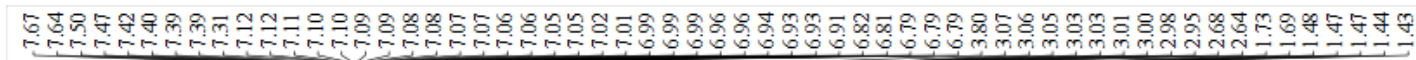

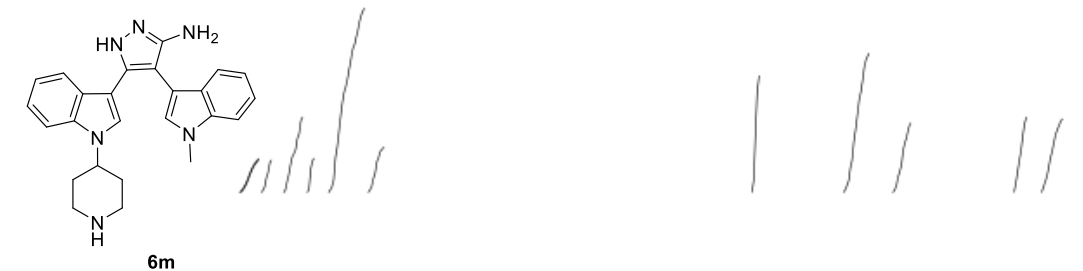

$6 m$

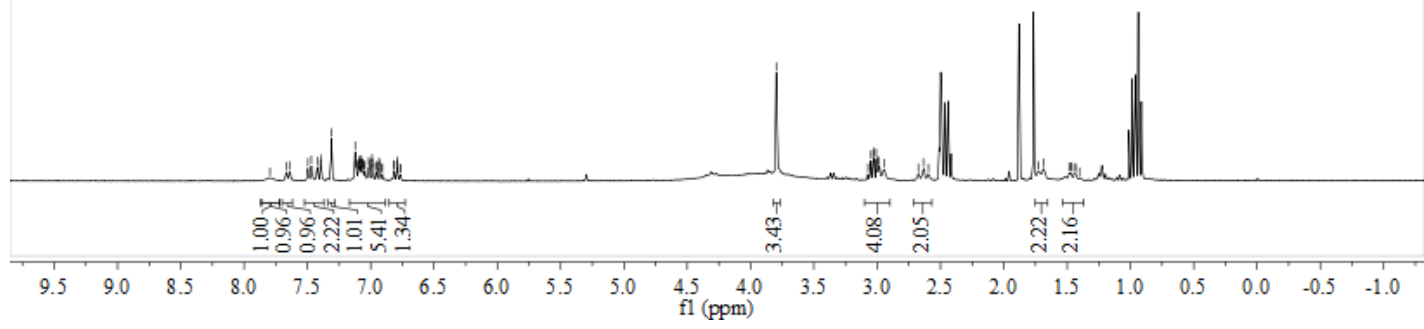

(7-(3-amino-4-(1-methyl-1H-indol-3-yl)-1H-pyrazol-5-yl)-3,4-dihydro-

[1,4]diazepino[6,7,1-hi]indol-2(1H)-yl)(piperidin-1-yl)methanone (7a).

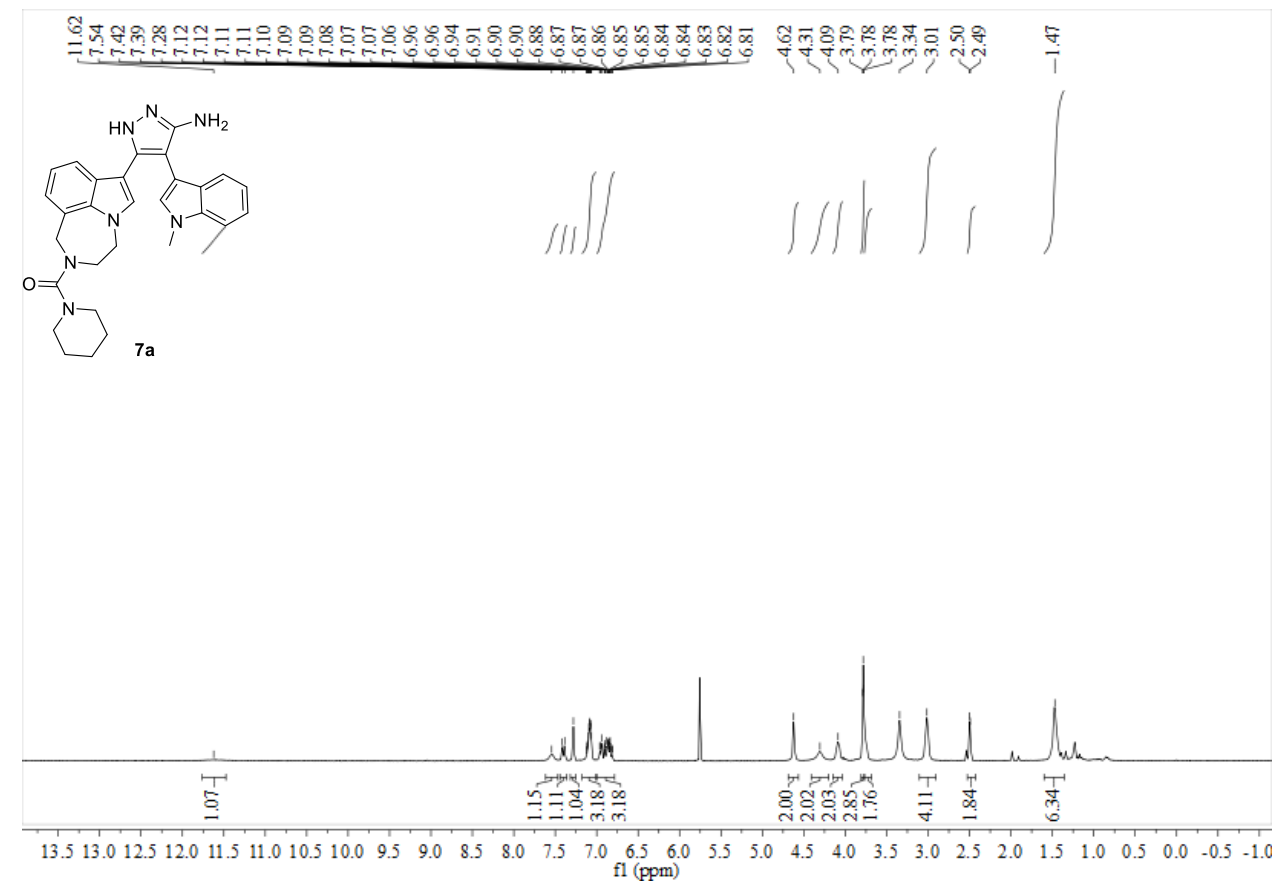




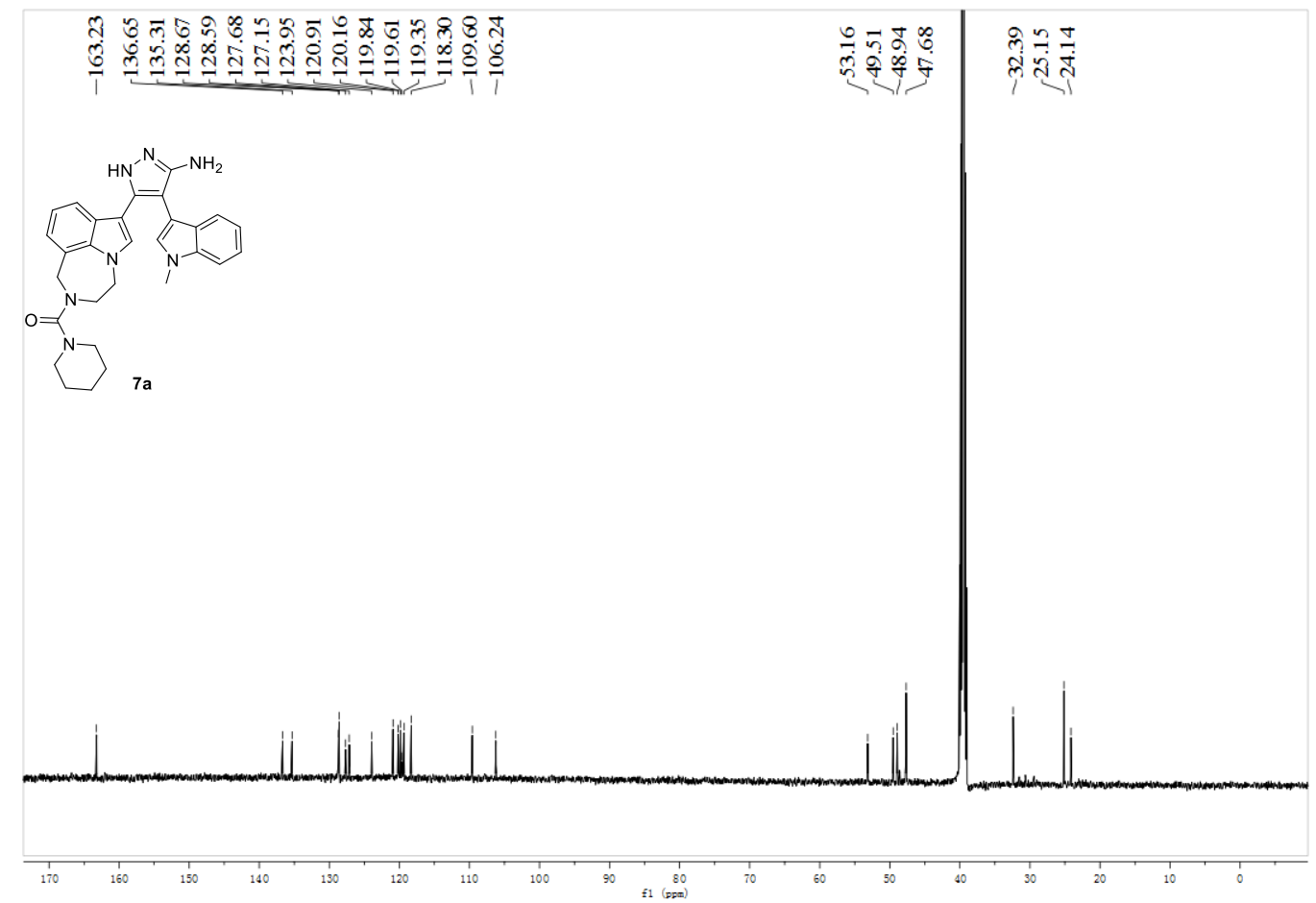

tert-butyl 7-(3-amino-4-(1-methyl-1H-indol-3-yl)-1H-pyrazol-5-yl)-3,4-dihydro[1,4]diazepino[6, 7,1-hi]indole-2(1H)-carboxylate(7b).

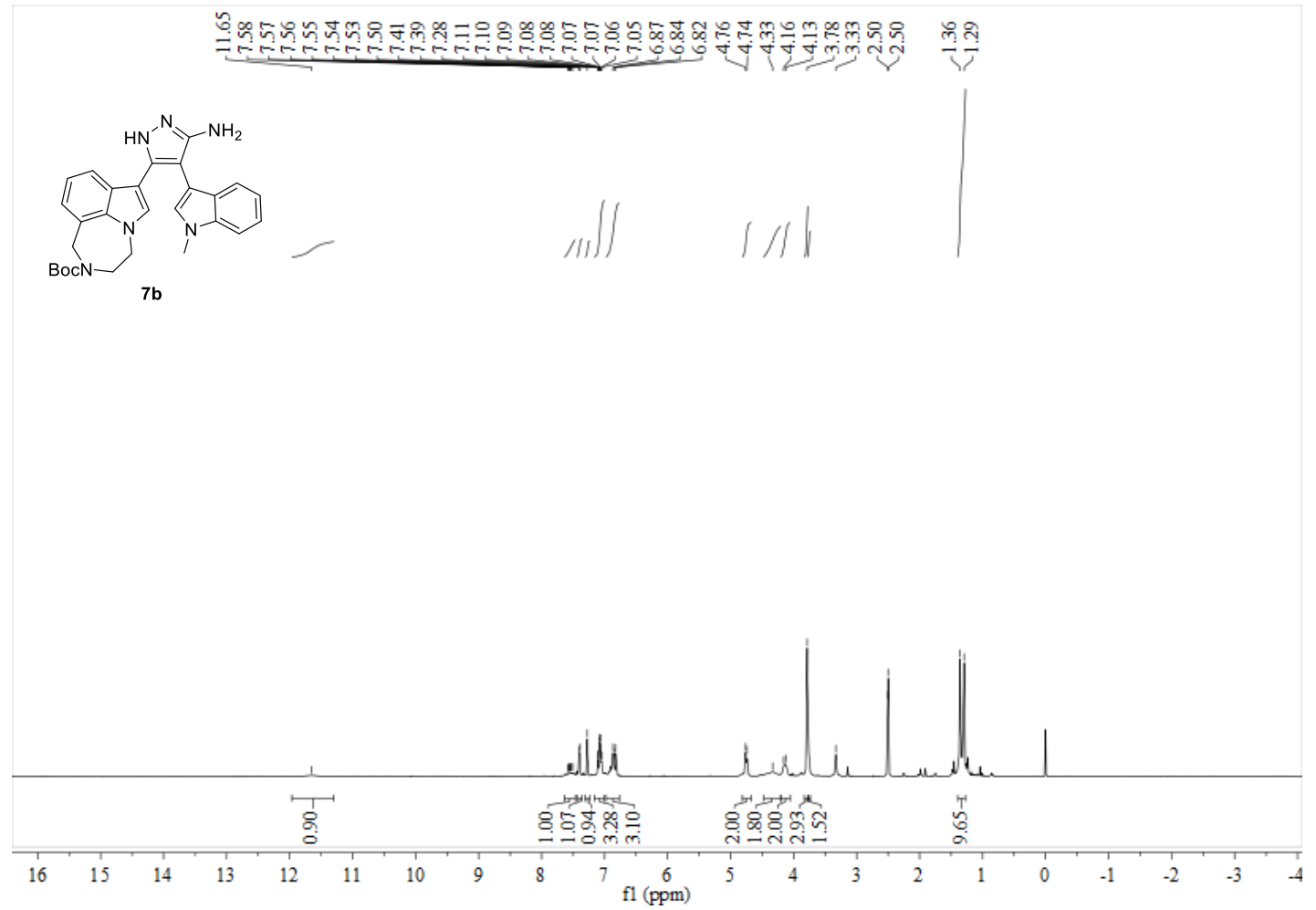




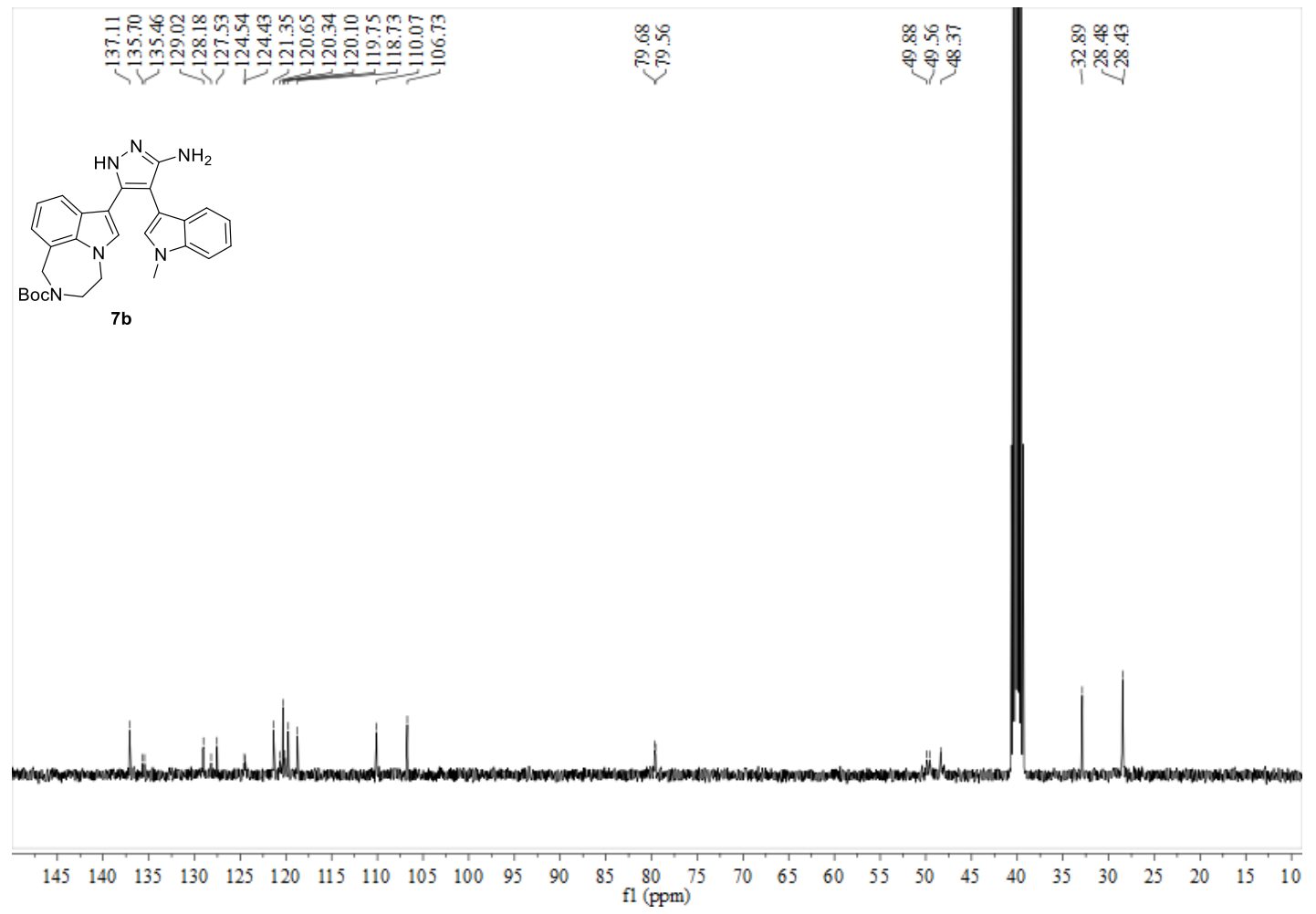

4-(1-methyl-1H-indol-3-yl)-5-(1,2,3,4-tetrahydro-[1,4]diazepino[6,7,1-hi]indol-7-yl)1H-pyrazol-3-amine(7c).

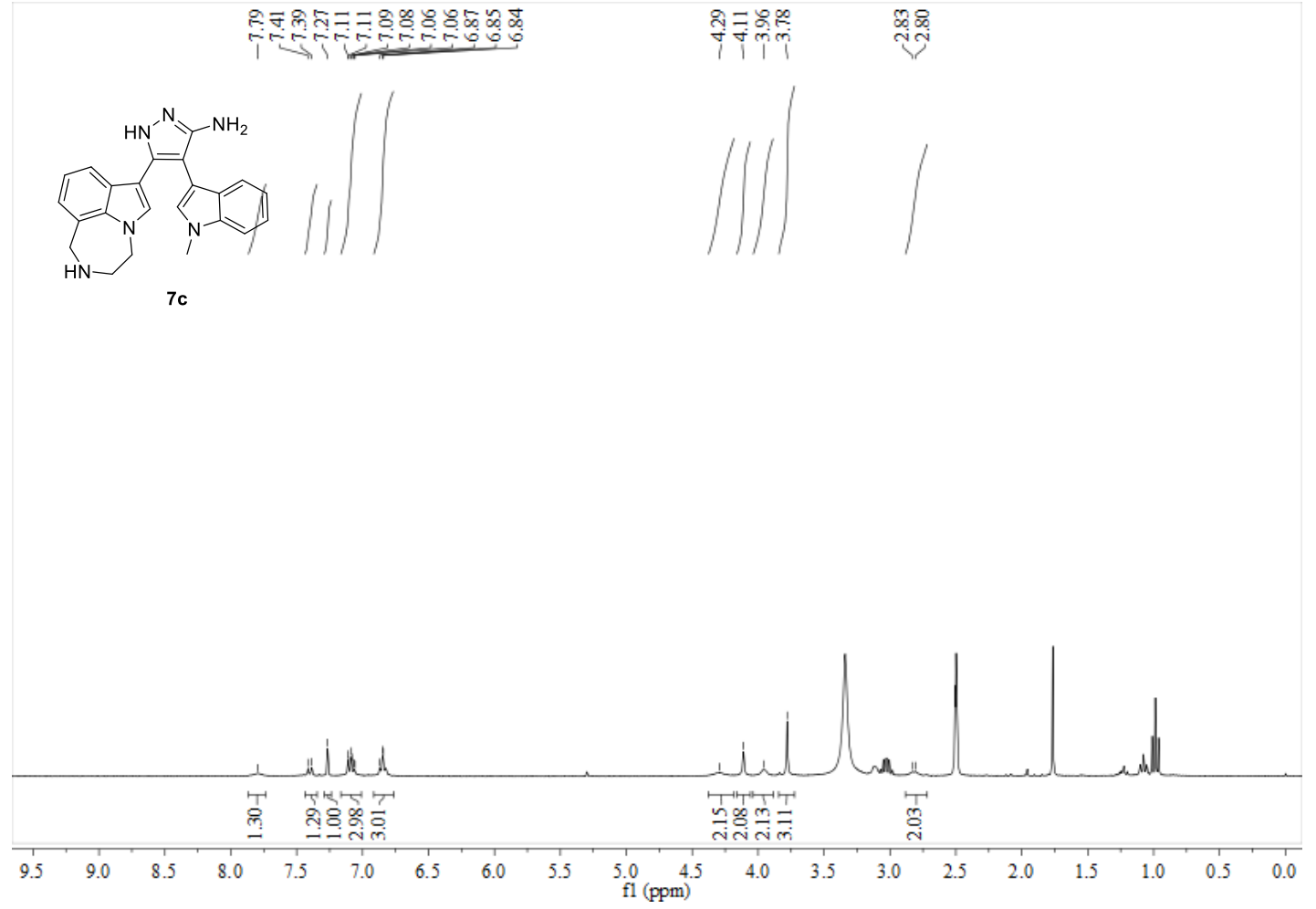


A

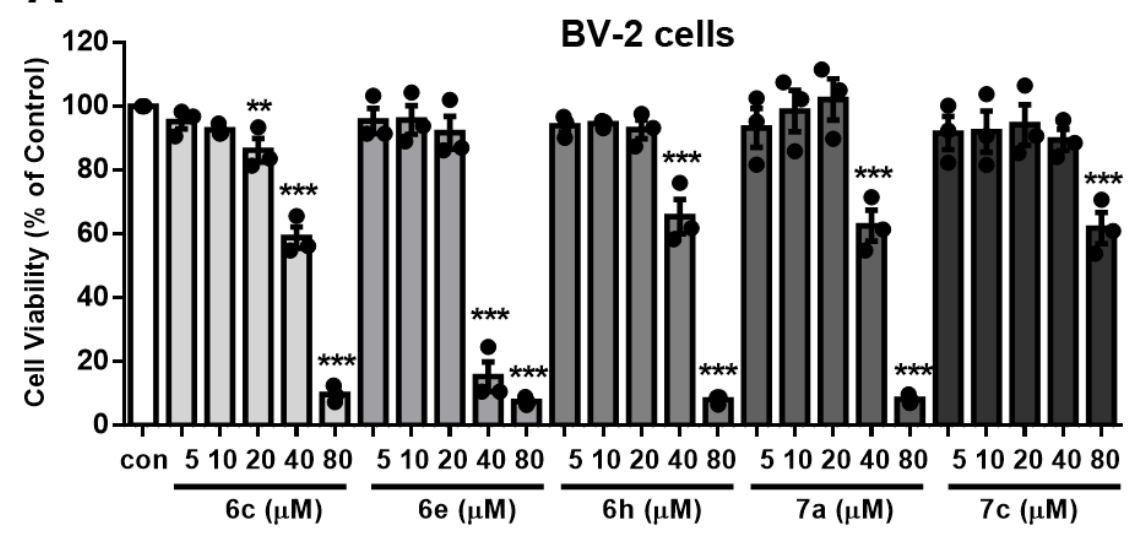

B

HT-22 cells

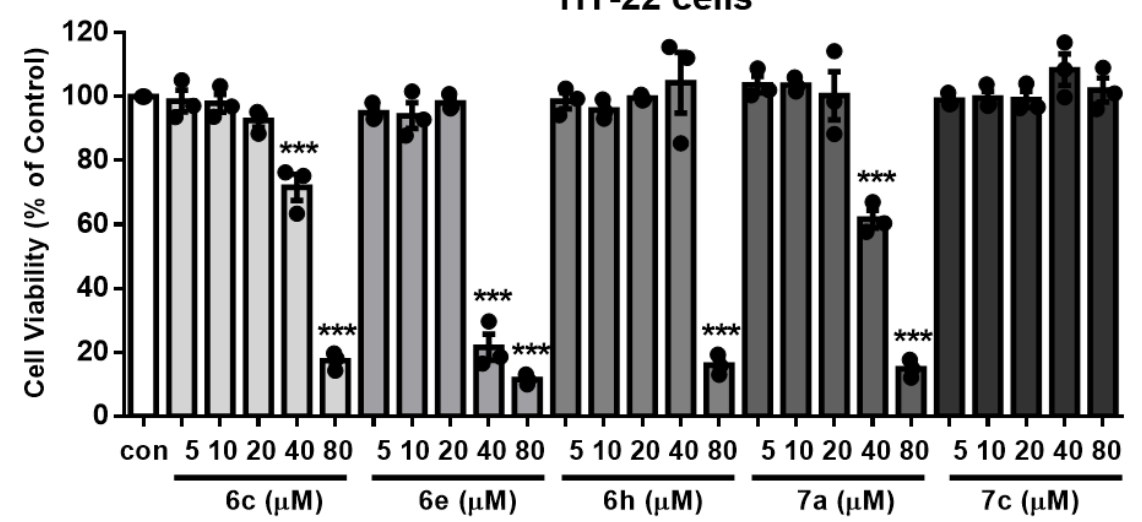

Figure S1. The cytotoxic effects of compounds $6 \mathbf{c}, \mathbf{6 e}, \mathbf{6 h}, \mathbf{7 a}$ and $7 \mathbf{c}$ on BV-2 cells (A) and HT-22 cells (B). Cell viabilities of BV-2 cells or HT-22 cells exposed to test compounds (5-80 $\mu \mathrm{M})$ for 24 $\mathrm{h}$ were determined by MTT assay. Vehicle-treated cells were used as controls. Data are presented as percentage of control group, and shown as the mean $\pm \operatorname{SEM}(n=3) . * * p<0.01, * * * p<0.001$ vs the control group (con). 